\title{
Episodic Liquidity Crises: Cooperative and Predatory Trading
}

\author{
BRUCE IAN CARLIN, MIGUEL SOUSA LOBO, and S. VISWANATHAN*
}

\begin{abstract}
We describe how episodic illiquidity arises from a breakdown in cooperation between market participants. We first solve a one-period trading game in continuous-time, using an asset pricing equation that accounts for the price impact of trading. Then, in a multi-period framework, we describe an equilibrium in which traders cooperate most of the time through repeated interaction, providing apparent liquidity to one another. Cooperation breaks down when the stakes are high, leading to predatory trading and episodic illiquidity. Equilibrium strategies that involve cooperation across markets lead to less frequent episodic illiquidity, but cause contagion when cooperation breaks down.
\end{abstract}

WhY IS ILLIQUIDITY RARE and episodic? Pastor \& Stambaugh (2003) detect only 14 aggregate low-liquidity months over the time period 1962 to 1999 . Despite being of significant magnitude, most of the episodes were short-lived and were followed by long periods of liquidity. ${ }^{1}$ The origin of this empirical observation still remains a puzzle. In this paper, we attempt to shed light on this puzzle by developing a theoretical model in which a breakdown in cooperation between traders in the market manifests itself in predatory trading. This mechanism leads to sudden and short-lived illiquidity.

We develop a dynamic model of trading based on liquidity needs. During each period, a liquidity event may occur in which a trader is required to liquidate a large block of an asset in a relatively short time period. This need for liquidity is observed by a tight oligopoly, whose members may choose to predate or cooperate. Predation involves racing and fading the distressed trader to the market,

\footnotetext{
*Bruce Ian Carlin is from the Anderson Graduate School of Management, University of California at Los Angeles. Miguel Sousa Lobo and S. Viswanathan are from the Fuqua School of Business at Duke University. The authors would like to thank Robert Almgren, Yacov Amihud, Kerry Back, Ravi Bansal, Michael Brandt, Markus Brunnermeier, Bhagwan Chowdry, Phil Dybvig, Simon Gervais, Milt Harris, Roy Henriksson, Ming Huang, Ron Kaniel, Arvind Krishnamurthy, Pete Kyle, Leslie Marx, Atif Mian, David Mordecai, Lasse Pedersen, Matt Pritsker, Uday Rajan, Ioanid Rosu, Ronnie Sadka, Louis Scott, Duane Seppi, Jim Smith, Matt Spiegel, Curt Taylor, Jiang Wang, Huseyin Yildirim, an anonymous referee, and seminar participants in the 2005 World Congress of Economics, the 2005 New York Federal Reserve Bank conference on liquidity, the 2006 Atlanta Federal Reserve conference on risk, the IAFE Liquidity Risk Symposium, the 2006 Mitsui Life Financial Center Symposium, the Duke Finance and Duke Economics seminars, and the Indian School of Business for their comments.

${ }^{1}$ See Acharya and Pedersen (2005) for evidence of flight to quality during these episodes.
} 
causing an adverse price impact for the trader. ${ }^{2}$ Cooperation involves refraining from predation and allows the distressed trader to transact at more favorable prices. In our model, traders cooperate most of the time through repeated interaction, providing "apparent liquidity" to one another. However, episodically this cooperation breaks down, especially when the stakes are high, which leads to opportunism and the loss of this apparent liquidity.

The following quote provides a recent example of an episodic breakdown in cooperation between two cooperative periods in the European debt market (New York Times, Sept. 15, 2004):

The bond sale, executed Aug. 2, caused widespread concern in Europe's markets. Citigroup sold 11 billion euros of European government debt within minutes, mainly through electronic trades, then bought some of it back at lower prices less than an hour later, rival traders say. Though the trades were not illegal, they angered other bond houses, which said the bank violated an unspoken agreement not to flood the market to drive down prices. ${ }^{3}$

This quote suggests that market participants generally cooperate, though there is episodic predation that leads to acute changes in prices. Note that predatory behavior can involve either exploiting a distressed trader's liquidity requirements or inducing another trader to be distressed.

There exists some empirical evidence that cooperation affects price evolution and liquidity in financial markets. Cooperation and reputation have been shown to affect liquidity costs on the floor of the New York Stock Exchange (NYSE). Battalio, Ellul, and Jennings (2005) document an increase in liquidity costs in the trading days surrounding a stock's relocation on the floor of the exchange. ${ }^{4}$ They find that brokers who simultaneously relocate with the stock and continue their long-term cooperation with the specialist obtain a lower cost of liquidity, which manifests in a smaller bid-ask spread. Cocco, Gomes, and Martins (2003) detect evidence in the interbank market that banks provide liquidity to each other in times of financial stress. They find that banks establish lending relationships in this market to provide insurance against the risk of shortage or excess of funds during the reserve maintenance period. ${ }^{5}$

We model the effects of cooperation and predation on liquidity as follows. We start by establishing a predatory stage game in continuous time, and then

\footnotetext{
${ }^{2}$ Brunnermeier and Pedersen (2005) define predatory trading as trading that induces and/or exploits another investor's need to change their position. It is important to distinguish predatory trading from front-running. Front-running is an illegal activity whereby a specialist, acting as an agent of an investor, trades on his own account in the same direction as his client before he fulfills his client's order. In this way, the specialist profits but violates his legal obligation as an agent of the investor. Predatory activity occurs in the absence of such a legal obligation.

${ }^{3}$ On February 2, 2005 the Wall Street Journal reported that this predatory trading plan was referred to as "Dr. Evil" by traders working at Citicorp.

${ }^{4}$ This is an exogenous event that changes long-run relationships between brokers and the specialist.

${ }^{5}$ Other papers in this literature include Berhardt et al. (2005), Desgranges and Foucault (2005), Reiss and Werner (2004), Ramadorai (2003), Hansch, Naik, and Viswanathan (1999), and Massa and Simonov (2003).
} 
model cooperation by embedding it in a repeated game framework. In the stage game, each trader faces a differential game with other strategic traders, in which trading has both a temporary and a permanent impact on the price of the asset. That is, the price of the asset is affected by the current rate of trading (temporary price impact) and by the total cumulative quantity traded over time (permanent price impact). ${ }^{6}$ Because we use a pricing equation that accounts for the effect of trading pressure on prices (in contrast to the model of Brunnermeier and Pedersen (2005)), in aggregate the strategic traders suffer a surplus loss in the presence of predatory trading. This surplus loss motivates the traders to cooperate and provide liquidity to each other in our repeated game. ${ }^{7,8}$

In the equilibrium of our stage game, traders race to market, selling quickly in the beginning of the period, at an exponentially decreasing rate. Also in equilibrium, predators initially race the distressed traders to market, but eventually fade them and buy back. This racing and fading behavior is well known in the trading industry and is modeled by Foster and Viswanathan (1996).

We model cooperation by embedding the predatory stage game in a dynamic game. We first consider an infinitely repeated game in which the magnitude of the liquidity event is fixed. In this framework, there exists an extremal equilibrium that is Pareto superior for the traders. We then extend the model to episodic illiquidity by allowing the exogenous magnitude of the liquidity event in the repeated game to be stochastic. Given such stochastic liquidity shocks, we provide predictions as to the magnitude of the liquidity event required to trigger liquidity crises and we describe how a breakdown in cooperation leads to price volatility. Finally, we allow for multimarket contact in the stochastic version of our dynamic game. This increases cooperation across markets, but leads to contagion of both predation and liquidity crises across markets. ${ }^{9}$

\footnotetext{
${ }^{6}$ Motivation for partitioning price effects based on permanent and temporary components of liquidity is given in the body of the paper, but is based on work by Kraus and Stoll (1972), Holthaisen et al. (1990), Cheng and Madhavan (1997), Huang and Stoll (1997), and Sadka (2005).

${ }^{7}$ In the formulation by Brunnermeier and Pedersen, no transaction costs are incurred in the equilibrium solution and all gains by the predators are exactly offset by losses by distressed traders, so that there would be no feasible Pareto improvement in a repeated game. There are other substantial differences between the models. Brunnermeier and Pedersen impose exogenous holding limits $[-\bar{x}, \bar{x}]$ for traders, whereas we do not make this restriction. Our model involves a stochastic price process, while in Brunnermeier and Pedersen the asset pricing relationship is deterministic. Finally, note that Brunnermeier and Pedersen's model predicts price-overshooting, whereas our model does not. However, this is a consequence of the fact that, in our stage game model, all traders have an identical time horizon. If we relax this assumption as in Brunnermeier and Pedersen to allow predators to have a longer horizon, price overshooting also obtains in our model.

${ }^{8}$ Attari et al. (2005) also describe predatory trading behavior with a two-period model. They show that predators may even lend to others that are "financially fragile" because they can obtain higher profits by trading against them for a longer period of time. Our model is more general in that it is in an infinite-horizon, multiperiod framework, with each period a continuous-time game.

${ }^{9}$ Our mechanism for contagion is different from that of Brunnermeier and Pedersen (2005). The contagion in Brunnermeier and Pedersen (2005) is caused by a wealth effect. As prices in the market drop, additional traders are induced into a state of distress and a market-wide sell-off arises. In contrast, contagion in our model occurs when traders abandon cooperation in a model of repeated interaction.
} 
We note a few empirical implications of our model. We show that the need for liquidity over time needs to be sufficiently symmetric for the traders to cooperate; asymmetric distress probabilities lead to the abandonment of cooperation in equilibrium. We also show that traders are more likely to cooperate in markets in which the permanent price impact of trading is high and the temporary price impact of trading is low. These markets are also those in which the predatory equilibrium strategies are the most aggressive. We would expect liquidity in these markets to be smooth most of the time and the episodes of illiquidity to be the most marked. If the degree of asymmetric information associated with an asset is a good predictor of the permanent price impact of trading, then securities that have concentrated ownership, that have high insider ownership, or that are from high-growth, research-intensive firms should exhibit this type of pattern and have the most marked spikes in illiquidity. In contrast, securities with diffuse ownership of shares from mature (value) firms should exhibit spikes in illiquidity with lower magnitude. To the extent that higher average daily trading volume and higher number of outstanding shares are good predictors of a lower temporary impact of trading, securities with a large number of outstanding shares that are traded in large volumes should also have more steady apparent liquidity, but more marked illiquidity episodes. To our knowledge, even though this empirical typology seems natural, securities have not been grouped into these categories before and studied for their permanent and transitory illiquidity, which would be required to test the implications of our model. ${ }^{10}$

The rest of the paper is organized as follows. Section I introduces the price equation and sets up the stage game. We derive closed-form solutions for the trading dynamics and quantify the surplus loss due to competitive trading. Section II uses the stage game with one predator and one distressed trader as the basis for a multiperiod game. In Section II.A, we consider an infinitely repeated game in which the magnitude of the liquidity shocks is fixed and provide a model for the relationship between insiders and outsiders in these markets. In Section II.B, we model episodic illiquidity by allowing the magnitude of the liquidity shocks to be stochastic and we address the contagion of illiquidity across markets. Section III concludes. Appendix A contains all proofs. The stage-game solution in Section I is based on the equilibrium over open-loop strategies; in Appendix B we consider the equilibrium over closed-loop strategies and argue that the results are not qualitatively different.

\section{Trading and Predation}

\section{A. Asset Price Model}

The economy consists of two types of participants. The first type are strategic traders, which we index with $i=1,2, \ldots, n$. These traders are risk-neutral and

\footnotetext{
${ }^{10}$ Another possible test of our model would be a natural experiment. For example, comparing the liquidity pattern in the foreign exchange market before and after electronic automation might reveal the effects of relationships and cooperation on liquidity.
} 
maximize trading profits. They form a tight oligopoly over order flow in the financial market. Large traders are usually present in markets as proprietary trading desks, trading both on their own accounts as well as for others. The strategic traders observe the order flow and have inside information regarding transient liquidity needs in the market. They attempt to generate profits through their ability to forecast price moves and affect asset prices.

The second type of players are long-term investors, who form the competitive fringe. The long-term investors usually trade in the interest of mutual funds or private clients and exhibit less aggressive trading strategies. Long-term investors are more likely to follow a buy and hold strategy, limit the number of transactions they undertake, and avoid taking over-leveraged positions. They trade according to fundamentals. The primary difference between the two types of traders is that the long-term investors are not aware of transient liquidity needs in the economy.

There exists a risk-free asset and a risky asset, traded in continuous time. The aggregate supply $S>0$ of the risky asset at any time $t$ is divided between the strategic investors' holdings $X_{t}$ and the long-term investors' holdings $Z_{t}$ such that $S=X_{t}+Z_{t}$. The return on the risky asset is stochastic. The yield on the risk-free asset is zero.

The asset is traded at the price

$$
P_{t}=U_{t}+\gamma X_{t}+\lambda Y_{t}
$$

where

$$
d X_{t}=Y_{t} d t
$$

and $U_{t}$ is the stochastic process $d U_{t}=\sigma\left(t, U_{t}\right) d B_{t}$, with $B_{t}$ some onedimensional Brownian motion on $(\Omega, \mathcal{F}, P)$.

A similar pricing relationship was previously derived by Vayanos (1998), and also by Gennotte and Kyle (1991) who show that it arises from the equilibrium strategies between a market maker and an informed trader when the position of the noise traders follows a smoothed Brownian motion. Pritsker (2004) obtains a similar relationship for the price impact of large trades when institutional investors transact in the market. Huberman and Stanzl (2004b) find a similar relationship in discrete time. ${ }^{11,12}$

The pricing equation has three parts. The first term, $U_{t}$, represents the expected value of future dividends and is modeled as a martingale stochastic

\footnotetext{
${ }^{11}$ Directly linking trading pressure and price distinguishes our model from Brunnermeier and Pedersen (2005), where transaction costs are modeled via an exogenous parameter $A$ (the maximum trading rate at which transaction costs are avoided), which does not directly affect prices in the market. This results in there being no surplus to be gained from cooperation.

${ }^{12}$ Further motivation for our use of this pricing relationship are the empirical and theoretical studies that link trading pressure and asset prices (Keim and Madhavan (1996), Kaul, Mehrotra, and Morck (2000), Holthausen, et al. (1990), Chan and Lakonishok (1995), Bertsimas and Lo (1998), Fedyk (2001), DeMarzo and Uroevic (2000), Almgren and Chriss (1999), and Huberman and Stanzl (2004a)).
} 
diffusion process. The diffusion does not include a drift term, which is justified by the short-term nature of the events modeled..$^{13}$

The second and third terms partition the price impact of trading into permanent and temporary components. Such a decomposition is justified on theoretical grounds, as we note above, and seems reasonable in light of the empirical work on block trades by Kraus and Stoll (1972), Holthausen, Leftwich, and Mayers (1990), and Cheng and Madhavan (1997), who find large permanent and temporary effects for block trades on the NYSE. Cheng and Madhavan (1997) estimate the temporary and permanent price impacts of block trades exceeding 10,000 shares in the "downstairs" and "upstairs" markets at the NYSE. The permanent price impact, measured from the price at the trade immediately prior to the block trade to the price of the 20th trade after the block trade, is -6.66 in the downstairs market and -7.59 in the upstairs market, in basis points of the logarithmic return averaged over blocks of all sizes. The temporary price impact, measured from the price at the 20th trade after the block trade to the price at which the block was traded, is -5.28 downstairs and -5.81 upstairs, again in basis points of the logarithmic return averaged over blocks of all sizes. Cheng and Madhavan show that both the permanent and temporary price impact of trading increase in magnitude with the size of the traded block. For instance, the permanent price impact in the downstairs market is -5.63 for blocks of size 10 to 20 (thousands), -8.77 for blocks of size 20 to 50 , and -15.09 for blocks over 50. Importantly, Sadka (2005) finds that the correlation between the temporary component and the permanent component is around 0.28 , which suggests that there is significant variation in the ratio of the temporary price impact to the permanent price impact. ${ }^{14}$

In the second term, $X_{t}=\sum_{i=1}^{n} X_{t}^{i}$ is the inventory variable, which measures the aggregate amount of the asset that the strategic traders hold at time $t$. As $X_{t}$ increases, the supply available to the long-term investors decreases and the price at which they can access the asset increases. The parameter $\gamma$ measures the permanent liquidity effects of trading, that is the change in the price of the asset that is independent of the rate at which the asset is traded. Note that the level of asymmetric information in an asset is likely to be a major determinant of $\gamma$, as demand for the asset will then play a more important role in price formation. For instance, we expect an AAA-rated corporate bond to have lower asymmetric information associated with it than a B-rated bond. In our

\footnotetext{
${ }^{13}$ In a model with no discounting, Huberman and Stanzl (2004a) show that the presence of a drift term is inconsistent with no arbitrage. This result can be extended to the case with discounting. For no arbitrage to hold, the difference between the drift coefficient and the continuous-time discount factor must be zero. For the multiperiod game that we discuss later, the assumption is that $T$ is relatively small, that is, the distress and predation events develop over short periods of time, and the discounting within each period is not significant. The period-to-period discount factor is then also close to one. Since each period is short, the multistage game consists of many short periods, where the probabilities of a player being distressed in any one of those periods are small, so that the period-to-period discount factor is significant to the problem.

${ }^{14}$ Huang and Stoll (1997) show large variation in the ratio $\frac{\gamma}{\lambda}$ in Table 2 in their paper. For the stocks considered, the ratio $\frac{\gamma}{\lambda}$ varies from 0.02 to 0.22 , which seems especially significant given the sample of large liquid stocks that they consider.
} 
model, the AAA-rated bond should then have a lower $\gamma$ than the B-rated bond. Likewise, an asset with concentrated ownership should have a higher level of asymmetric information (and therefore a higher $\gamma$ ) than an asset with a more dispersed ownership structure. For an asset with more asymmetric information, the market will more strongly adjust the asset price based on the net change in the supply of the asset.

The third term, $Y_{t}=\sum_{i=1}^{n} Y_{t}^{i}$, measures the instantaneous, reversible price pressure that results from trading. The faster the traders sell, the lower a price they will realize. This leads to surplus loss effects, which we discuss in the following subsections. The price impact parameter $\lambda$ measures the temporary, reversible asset price change that occurs during trading. Trading volume and shares outstanding are likely to play a role in the level of $\lambda$.

\section{B. Stage Game: Trading Dynamics}

Our stage game is one of complete information. Many assets that are prone to illiquidity are traded in nonanonymous markets in which a few large dealers dominate order flow. ${ }^{15}$ Furthermore, roughly half of the trading volume at the NYSE is traded in blocks over 10,000 shares (Seppi (1990)) and much of that occurs in the upstairs market, which is nonanonymous. As a result, the liquidity needs of large traders are usually observed quickly by others.

For an example of a context in which this game structure is a natural choice, consider a thinly traded corporate bond issue that is traded by a small number of broker-dealers. Trading occurs either by direct negotiation over the phone, or by "sunshine trading" in which a mini-auction is held. The players are well known to each other because each deals repeatedly with the others. Their trading habits and strategies are common knowledge. ${ }^{16}$ When one trader needs to trade a large block of shares of an asset, this need is observed by others in the market and the optimal trading strategies solve a game of complete information.

In the stage game, strategic traders are either distressed or they are predators. A liquidity event occurs at time $t=0$, whereby the distressed traders are required to buy or sell a large block of the asset $\Delta x$ in a short time horizon $T$ (say, by the end of the trading day). Forced liquidation usually arises because of the need to offset another cash-constrained position such as an overleveraged position, or it occurs as a result of a risk management maneuver. The predators are informed of the trading requirement of the distressed traders and compete strategically in the market to exploit the price impact of the distressed traders' selling. For clarity of exposition, we assume that the opportunistic traders must return to their original positions in the asset by the end of the trading period,

\footnotetext{
${ }^{15}$ Duffie, Garleanu, and Pedersen (2005) also study the factors that affect liquidity in nonanonymous markets.

${ }^{16}$ A particularly clear example of this is the mortgage market developed by Salomon Brothers. Once this market was established and profitable, many of Salomon's mortgage traders were hired by other investment banks to run their mortgage desks. As a consequence, the trading habits of all of the desks were especially well known to each other.
} 
and that the distressed traders are informed of this requirement. ${ }^{17}$ Furthermore, we assume that, except for their trading targets, all strategic traders are identical. That is, the only difference between the two types of strategic traders is that the trading target is $\Delta x$ for the distressed traders, and zero for the predatory traders.

At the start of the stage game, every trader chooses a trading schedule $Y_{t}^{i}$ over the period $[0, T]$ to maximize their own expected value, assuming the other traders will do likewise. Subject to their respective initial and terminal holding constraints, they solve the dynamic program

$$
\begin{array}{cl}
\underset{Y_{t}^{i} \in \mathcal{Y}}{\operatorname{maximize}} & \mathbf{E}\left[\int_{0}^{T}-P_{t} Y_{t}^{i} d t\right] \\
\text { subject to } & X_{0}^{i}=x_{0 i} \\
& X_{T}^{i}=x_{T i},
\end{array}
$$

where the maximization is over $Y_{t}^{i}, t \in[0, T]$, with the strategy space $\mathcal{Y}$ restricted so that, for each $i, Y_{t}^{i}$ is continuous and admissible, that is, it satisfies the integrability condition

$$
\mathbf{E}\left[\int_{0}^{T}-P_{t} Y_{t}^{i} d t\right]<\infty .
$$

Note that $P_{t}$ depends on $Y_{t}^{i}$ and on the trading strategies of other traders as in equation (1). The restriction $Y_{t}^{i} \in L^{2}$, (i.e., $\int_{0}^{T}\left(Y_{t}^{i}\right)^{2}<\infty$ ), among others, ensures admissibility. It follows that the position $X_{t}^{i}$ is a differentiable function with continuous derivatives. ${ }^{18,19}$

We restrict our analysis in the paper to the open-loop Nash equilibrium solutions to this differential game. ${ }^{20}$ In equilibrium, each trader chooses ex ante a

\footnotetext{
${ }^{17}$ A variation on this model would be to allow the opportunistic traders to trade over a longer time period than the distressed traders. The solution for such a model is similar. However, the predatory trader will now choose what position to have by the end of the distressed seller's deadline. The choice of this position is made by maximizing the expected value from trading over the distressed seller's period, plus the expected value from selling the position at the end of that period at a constant rate over the additional time. (See also Footnote 7.)

${ }^{18}$ Our admissibility restriction is similar in spirit to Back and Baruch (2004). It restricts trader $i$ 's strategy given the price process, hence it depends on the strategies of other traders. As noted, it is easy to show that the restriction $Y_{t}^{i} \in L^{2}$ suffices to ensure admissibility, so that there are standard restrictions that ensure admissibility.

19 The single-trader version of this problem is related to Huberman and Stanzl (2004a,b), Almgren and Chriss (1999), and Bertsimas and Lo (1998).

${ }^{20}$ In Appendix B we analyze closed-loop strategies, where players take into account the other players' response functions and are able to change their trading schedules part-way through the game. From an asymptotic approximation (and numerical experiments), we find that the solution to the closed-loop differential game is qualitatively similar to that of the open-loop differential game, except that the welfare loss in the closed loop game is higher. Hence, the incentives for cooperation are stronger in the closed-loop solution, which would make the players more likely to cooperate in the repeated-game analysis of Section II.
} 
time-dependent trading strategy that is the best response to the other traders' expected actions. As we note in Appendix A, the restriction to smooth functionals that are admissible and the concavity of the objective function in $X_{t}^{i}$ and $Y_{t}^{i}$ ensures that the problem is well posed. ${ }^{21}$

The equilibrium solution of the open-loop problem is weakly time consistent. That is, the solution to the subproblem over the interval $\left[t_{1}, T\right]$ (with initial conditions as given by the solution of the [0,T]-problem at time $t_{1}$ ) is the truncation of the $[0, T]$-solution over that sub-interval. ${ }^{22}$ Along the equilibrium path, a trader follows a continuation strategy that maximizes his utility given his equilibrium play until that point. ${ }^{23}$

The following result outlines the unique Nash equilibrium solution for the traders. This formulation will serve as a basis for deriving the equilibrium strategies when several distressed traders are present without opportunism and when there are both opportunistic and distressed traders present in the economy. It will also allow for analysis of trader surplus, which motivates cooperation between traders in the repeated game.

RESUlT 1 (general solution): Consider $N$ traders that choose a time-dependent trading rate $Y_{t}^{i}$ to solve the optimization problem in Equation (3), subject to the asset price given by equation (1). The unique open-loop Nash equilibrium (among the class of continuous admissible functionals) in this game is for trader $i$ to trade according to

$$
Y_{t}^{i}=a e^{-\frac{n-1}{n+1} \frac{\gamma}{\lambda} t}+b_{i} e^{\frac{\gamma}{\lambda} t},
$$

with $a \in \mathbf{R}$, and $b_{i} \in \mathbf{R}, i=1, \ldots, n$, such that $\sum_{i=1}^{n} b_{i}=0$. The coefficients $a$ and $b_{i}$ are uniquely determined from the boundary conditions as follows

$$
\begin{aligned}
& a=\frac{n-1}{n+1} \frac{\gamma}{\lambda}\left(1-e^{-\frac{n-1}{n+1} \frac{\gamma}{\lambda} T}\right)^{-1} \frac{\sum_{i=1}^{n} \Delta x_{i}}{n} \\
& b_{i}=\frac{\gamma}{\lambda}\left(e^{\frac{\gamma}{\lambda} T}-1\right)^{-1}\left(\begin{array}{c}
\sum_{j=1}^{n} \Delta x_{j} \\
\Delta x_{i}-\frac{1}{n}
\end{array}\right),
\end{aligned}
$$

where $\Delta x_{i}=x_{T i}-x_{0 i}$.

\footnotetext{
${ }^{21}$ Our approach to existence is similar to Huberman and Stanzl (2004a), who consider a related problem in continuous time for a single trader. As in their paper, we restrict ourselves to the class of smooth differentiable strategies. An alternative approach is to ensure smoothness by defining the solution to the continuous-time problem as the limit of the solutions to a sequence of discrete-time problems, which is the approach we use in the analysis of the closed-loop version of the problem in Appendix B.

${ }^{22}$ See Theorem 6.12 in Basar and Olsder (1999) and the related discussion.

${ }^{23}$ However, the open-loop strategy is not strongly time consistent or subgame perfect. The closedloop solution discussed in Appendix B is strongly time consistent.
} 
The equilibrium trading strategy in equation (5) consists of two parts. For small $t$, the first term dominates the trading strategy and for larger $t$ within the interval $T$, the second term dominates. The first term, $a e^{-\frac{n-1}{n+1} \frac{\gamma}{\lambda} t}$, describes how fast traders race to the market during a sell-off or a buying frenzy. The second term, $b_{i} e^{\frac{\gamma}{\lambda} t}$, describes the magnitude of fading by each trader. Fading refers to traders reversing the direction in which they are trading and, for the cases we consider in our stage game, only occurs when opportunistic traders are present. For example, consider the case in which a distressed trader needs to sell a block of shares of an asset and predatory traders are present in the market. The first term describes the rate at which they all trade when they initially race each other to the market, and the second term describes the trading dynamic when the predators buy back.

Note that the constant $a$ in equation (6) is a function of the average trading target over all traders. All traders race to the market in similar fashion, based on the common knowledge of their overall trading target. Toward the end of the period, traders fade based on their particular trading targets. The constants $b_{i}$ are a function of how each trader's trading target is different from the average. A trader that is distressed, in the sense that he has a higher-than-average trading target, toward the end of the period will trade in the same direction as the racing. A trader that is a predator, in the sense that she has a smaller-thanaverage trading target, will fade in the opposite direction, that is, reverse the direction in which she is trading.

To develop more intuition regarding Result 1, we evaluate equation (5) for special cases, which we will use when we consider the games of repeated interaction in Section II.

CASE 1 (symmetric distressed traders): First, consider the optimal trading policy when a trader has monopoly power and buys or sells in the absence of other strategic traders. For $n=1$, the optimal trading policy (5) for a single trader is to trade at a constant rate, $Y_{t}=a=\frac{\Delta x}{T}$, where $\Delta x$ is the block of shares that the trader needs to buy or sell. This result is consistent with Bertsimas and Lo (1998) and Huberman and Stanzl (2004b).

Now, consider $n$ symmetric traders, each needing to sell an identical amount of shares $\frac{\Delta x}{n}$. From equation (5), the unique equilibrium smooth trading strategy is

$$
Y_{t}^{i}=a e^{-\frac{n-1}{n+1} \frac{\gamma}{\lambda} t}, \quad i=1, \ldots, n,
$$

where $a$ is as in equation (6) with $\sum_{i=1}^{n} \Delta x_{i}=\Delta x$. Figure 1 plots these trading policies with $\Delta x=1, T=1, \gamma=10$, and $\lambda=1$. If the permanent price impact of trading is lower (small $\gamma$ ), trading will occur comparatively later. If the temporary price impact of trading is smaller (small $\lambda$ ), trading will occur comparatively earlier. For $n=1$ we obtain the constant selling rate (solid horizontal line). If there are more traders, everybody will trade earlier. Note that the rate of trading goes to $e^{-\frac{\gamma}{\lambda} t}$ as $n \rightarrow \infty$ (dotted line). That is, there is an upper bound 


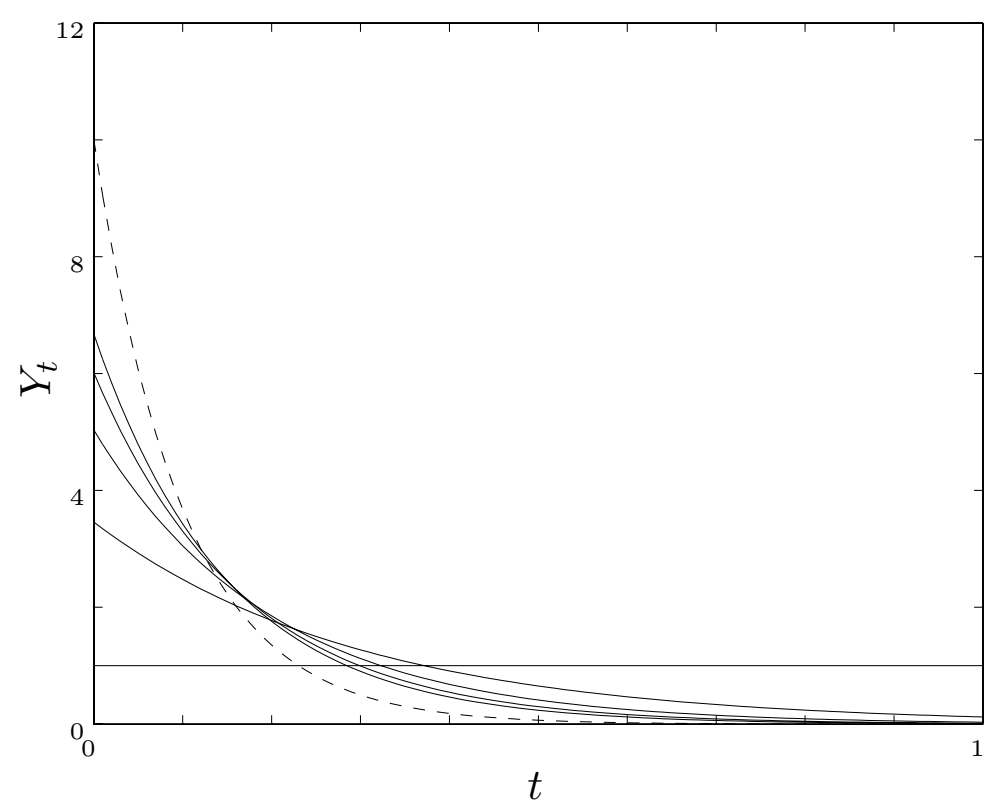

Figure 1. Trading rate $\left(Y_{t}\right)$ for multiple traders with identical targets (solid for $n=1$, $\mathbf{2 , 3}, \mathbf{4}, \mathbf{5}$, dashed for $\boldsymbol{n}=\infty$ ) during a market sell-off. Competition among traders leads to a race to trade. Traders sell at a decreasing exponential rate. For $n=1$ we obtain the constant selling rate (solid horizontal line). If there are more traders, everybody will trade earlier. Note that there is an upper bound on how fast traders will sell their position $\left(Y_{t} \rightarrow e^{-\frac{\gamma}{\lambda} t}\right.$ as $\left.n \rightarrow \infty\right)$, regardless of how many traders are in the race (dotted line). The parameters for this example are $\Delta x=1, T=1, \gamma=10$, and $\lambda=1$.

on how fast traders will sell their position, regardless of how many traders are in the race. Note that the shape of the curve depends on $\gamma$ and on $\lambda$ only through the ratio $\frac{\gamma}{\lambda}$. However, the scale of the $\gamma$ and $\lambda$ parameters matters in relation to $U_{t}$. For instance, the expected long-term fractional loss in the value of the asset is proportional to $\gamma / U_{t}$.

Figure 2 plots the corresponding price process (for a constant $U_{t}=50$ ). For a single trader, the price changes linearly over the trading period. By trading at a constant rate, the single trader is able to "walk down the demand curve" and not incur a loss in surplus due to excessive short-term price pressure from the trading intensity (straight solid line). For a large number of traders, the price function over $t \in[0, T]$ quickly approaches a constant value. The information regarding the trader's target position in the asset quickly becomes incorporated in the asset price (dotted line). There is a surplus loss to the strategic traders as trading pressure depresses prices quickly. We quantify these surplus changes in the next subsection.

CASE 2 (distressed trader and predatory trader): We now set up and analyze the two-player predatory stage game, which forms the basis for the games in 


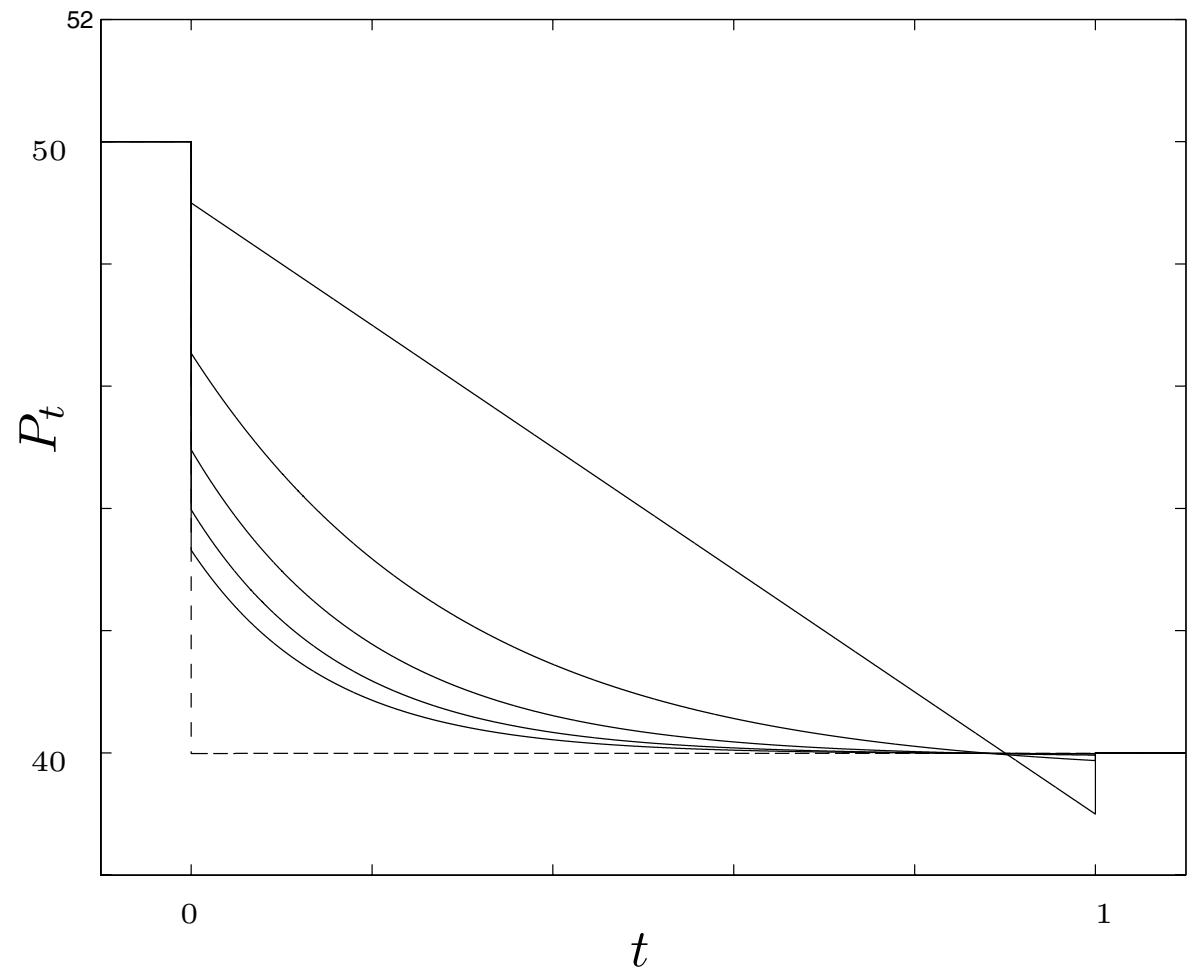

Figure 2. Expected price for multiple traders with identical targets (solid for $n=1,2$, $\mathbf{3}, \mathbf{4}, \mathbf{5}$, dashed for $\boldsymbol{n}=\infty$ ). When $n=1$, trading occurs at a constant rate (straight solid line). When there are a large number of traders ( $n$ large), trading pressure depresses prices quickly (dotted line). The parameters for this example are $\Delta x=1, T=1, \gamma=10$, and $\lambda=1$, with constant $U_{t}=50$.

Section II. Let there be one distressed and one opportunistic trader. Each trader chooses a trading schedule $\left(Y_{t}^{d}\right.$ and $\left.Y_{t}^{p}\right)$ over the period $[0, T]$ to maximize their own expected value, assuming the other trader will do likewise. From Result 1, the unique equilibrium smooth trading policies are

$$
\begin{aligned}
& Y_{t}^{d}=a e^{-\frac{1}{3} \frac{\gamma}{\lambda} t}+b e^{\frac{\gamma}{\lambda} t} \\
& Y_{t}^{p}=a e^{-\frac{1}{3} \frac{\gamma}{\lambda} t}-b e^{\frac{\gamma}{\lambda} t},
\end{aligned}
$$

where

$$
a=\frac{\gamma}{6 \lambda}\left(1-e^{-\frac{1}{3} \frac{\gamma}{\lambda} T}\right)^{-1} \Delta x, \quad b=\frac{\gamma}{2 \lambda}\left(e^{\frac{\gamma}{\lambda} T}-1\right)^{-1} \Delta x .
$$

The shape of the trading strategy depends on the parameters of the market. Figure 3 gives an example, with $\Delta x=1, T=1, \gamma=10$, and $\lambda=1$. The strategy involves the opportunistic trader initially racing the distressed trader to the market in an exponential fashion, and then fading the distressed trader toward 


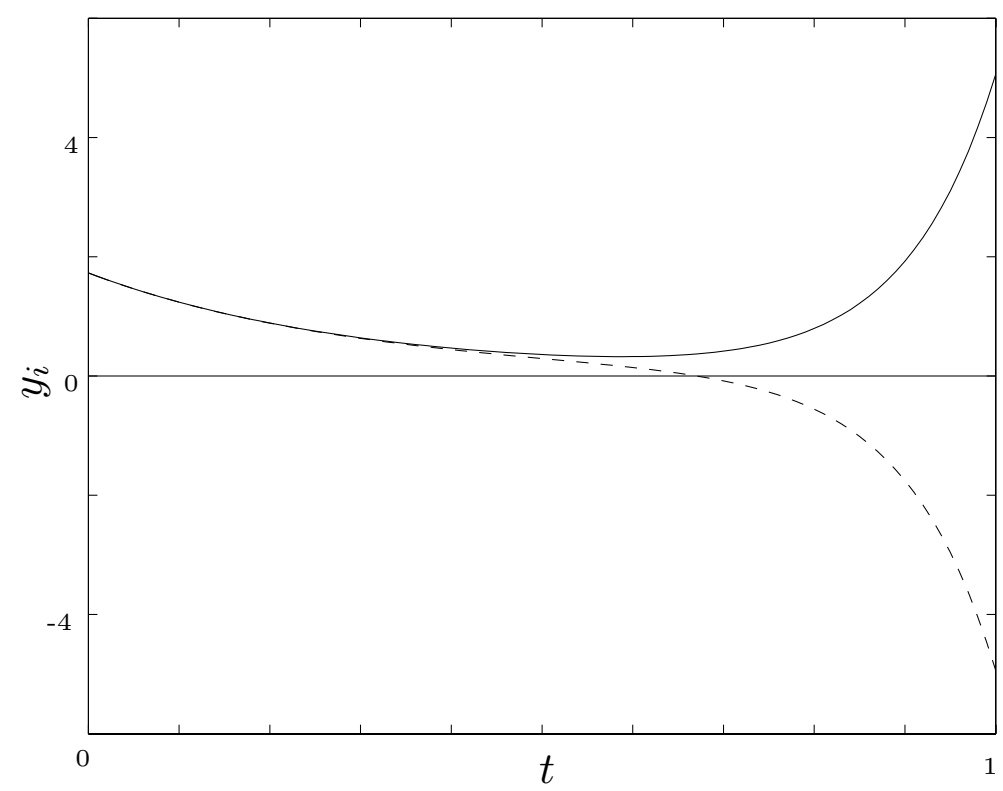

Figure 3. One trader with a position target (solid) and one opportunistic trader (dashed). The opportunistic trader initially races the distressed trader to the market in a selloff. Then, toward the end of the period, the opportunistic trader reverses position and fades the distressed trader. The parameters for this example are $\Delta x=1, T=1, \gamma=10$, and $\lambda=0.1$.

the end of the period, also exponentially. If the first trader needs to sell, that is, $\Delta x<0$, the predatory trader sells short initially and buys back in later periods to cover his position. If the distressed trader is required to buy a block of the asset, the predator follows the opposite strategy. In general, we see that the presence of the predator will lead the distressed trader to increase his trading volume at the beginning and at the end of the trading period.

\section{Surplus Effects}

Based on the trading dynamics in Section I.B, we quantify the surplus changes that occur when traders race to market and when predatory trading occurs. The surplus values that we derive will be used in the following sections.

First, consider the expected value for a single trader with monopoly power. Given the price (1) and the optimal trading rate $Y_{t}=\frac{\Delta x}{T}$, the expected value for the single trader is given by

$$
V_{1}=-U_{0} \Delta x-\left(\frac{\gamma}{2}+\frac{\lambda}{T}\right) \Delta x^{2} .
$$

This is the trader's first best when no other competing traders are informed of the trader's trading requirement $\Delta x$. The costs due to short-term trading 
pressure are minimized. When other players trade strategically at the same time, the value that the trader can derive is strictly lower than $V_{1}$. We will also see that when multiple traders compete in a sell-off or if there is predatory trading, the aggregate surplus available to all traders is lower.

Define $V_{n}$ as the total expected value for the strategic traders when $n$ traders play this game and define $\Delta V_{n}$ as the change in total surplus that occurs compared to the expected value when all participants trade at a constant rate $\left(V_{1}\right)$. The following result provides expressions for $V_{n}$ and $\Delta V_{n}$, and shows that the loss in surplus is increasing in the number of traders. This result lays some of the groundwork for the surplus results for the case in which there is one distressed and one predatory trader (i.e., for $n=2$ ), and is also of interest on its own for the monotonicities.

RESUlt 2 (expected total surplus and loss for multiple traders): The total expected value for $n$ traders with a combined trading target $\Delta x$ is

$$
V_{n}=-U_{0} \Delta x-\frac{\gamma}{2}\left(1+\frac{n-1}{n+1} \cdot \frac{e^{\frac{n-1}{n+1} \frac{\gamma}{\lambda} T}+1}{e^{\frac{n-1}{n+1} \frac{\gamma}{\lambda} T}-1}\right) \Delta x^{2} .
$$

The expected loss in total surplus from competition is

$$
\Delta V_{n}=V_{1}-V_{n}=\gamma\left(\frac{1}{2} \cdot \frac{n-1}{n+1} \cdot \frac{e^{\frac{n-1}{n+1} \frac{\gamma}{\lambda} T}+1}{e^{\frac{n-1}{n+1} \frac{\gamma}{\lambda} T}-1}-\frac{1}{\frac{\gamma}{\lambda} T}\right) \Delta x^{2}
$$

$\Delta V_{n}$ is positive, monotonically increasing in $\gamma, T$, and $n$ and monotonically decreasing in $\lambda$.

Now we apply Result 2 to the two-trader case and derive a surplus result that we use in Section II. Define $V_{2}$ as the total expected value for the strategic traders when two traders play this game, and define $V_{d}$ and $V_{p}$ as the expected values to the distressed trader and to the opportunistic trader (as defined in Section I.B). Likewise, define $\Delta V_{2}$ as the change in surplus that occurs compared to the expected value $V_{1}$ that is obtained when the participants trade at a constant rate.

RESUlt 3 (expected total surplus and loss for two traders): The total expected value for the distressed trader and the predatory trader is

$$
V_{2}=V_{d}+V_{p}=-U_{0} \Delta x-\frac{\gamma}{3} \cdot \frac{2 e^{\frac{1}{3} \frac{\gamma}{\lambda} T}-1}{e^{\frac{1}{3} \frac{\gamma}{\lambda} T}-1} \Delta x^{2} .
$$


The expected value is decomposed as

$$
\begin{aligned}
& V_{d}=-U_{0} \Delta x-\frac{\gamma}{6} \frac{5 e^{\frac{\gamma}{\lambda} T}+e^{\frac{2}{3} \frac{\gamma}{\lambda} T}+e^{\frac{1}{3} \frac{\gamma}{\lambda} T}-1}{e^{\frac{\gamma}{\lambda} T}-1} \Delta x^{2}, \\
& V_{p}=\frac{\gamma}{6} \cdot \frac{e^{\frac{2}{3} \frac{\gamma}{\lambda} T}-1}{e^{\frac{2}{3} \frac{\gamma}{\lambda} T}+e^{\frac{1}{3} \frac{\gamma}{\lambda} T}+1} \Delta x^{2} .
\end{aligned}
$$

The expected loss due to predation for the distressed trader is

$$
\Delta V_{d}=V_{1}-V_{d}=\gamma\left(\frac{1}{6} \cdot \frac{2 e^{\frac{\gamma}{\lambda} T}+e^{\frac{2}{3} \frac{\gamma}{\lambda} T}+e^{\frac{1}{3} \frac{\gamma}{\lambda} T}+2}{e^{\frac{\gamma}{\lambda} T}-1}-\frac{1}{\frac{\gamma}{\lambda} T}\right) \Delta x^{2},
$$

and the expected total surplus loss from predation for the strategic traders is

$$
\Delta V_{2}=V_{1}-V_{2}=\gamma\left(\frac{1}{6} \cdot \frac{e^{\frac{1}{3} \frac{\gamma}{\lambda} T}+1}{e^{\frac{1}{3} \frac{\gamma}{\lambda} T}-1}-\frac{1}{\frac{\gamma}{\lambda} T}\right) \Delta x^{2}
$$

$V_{p}$ is monotonically increasing in $\gamma$ and in $T$, and monotonically decreasing in $\lambda$.

The ratio of gains to the predator to the losses to the distressed trader, $\frac{V_{p}}{\Delta V_{d}}$, is monotonically decreasing in $\gamma$ and $T$, monotonically increasing in $\lambda$, and bounded by $\frac{4}{5}>\frac{V_{p}}{\Delta V_{d}}>\frac{1}{2}$. Note that it follows from Result 2 that $\Delta V_{2}$ is positive, monotonically increasing in $\gamma$ and $T$, and monotonically decreasing in $\lambda$. From the monotonicity of $V_{p}$ it also follows that $\Delta V_{d}=\Delta V_{2}+V_{p}$ is monotonic.

\section{Proof: See Appendix A. Q.E.D.}

From the solutions for the rate of trading, we can see that a larger $\frac{\gamma}{\lambda}$ ratio (more permanent price impact and less temporary price impact) creates conditions for more aggressive predation, in the sense that trading will be relatively more concentrated at the beginning and at the end of the period. Racing is faster and fading occurs closer to the end of the trading period.

Since $\frac{V_{p}}{\Delta V_{d}}$ is bounded in the interval $\left[\frac{1}{2}, \frac{4}{5}\right]$, the losses to the distressed trader are strictly higher than the gains by the predator. ${ }^{24}$ Even though the monotonicity of $V_{p}$ implies that market conditions that lead to more aggressive predation (larger $\gamma$, lower $\lambda$, or both) will lead to more gains from predation, since $\frac{V_{p}}{\Delta V_{d}}$ decreases in $\gamma$ and increases in $\lambda$, the losses to the distressed trader grow faster than the gains to the predator. In this one-shot stage game, this represents a significant surplus loss to the traders as a whole. ${ }^{25}$ In a dynamic setting, which we model in the next section, if both traders have a possible liquidity need

\footnotetext{
${ }^{24}$ Note that these bounds are tight, in that they can be approximated arbitrarily close under some combination of valid parameters.

${ }^{25}$ In our stage game, we do not allow for ex post renegotiation. Surplus losses are common in many models in noncooperative game theory (i.e., Prisoner's dilemma and centipede game) and motivate cooperation in repeated play.
} 
in each period, there exists a potential for Pareto improvement if the traders can cooperate. As we will see, the ratio $\frac{\gamma}{\lambda}$ is the key determinant of whether cooperation is possible.

Finally, for some insight into the magnitude of the available Pareto improvement, consider the case in which $\lambda \ll \gamma T$. Under this condition, predation is most aggressive, that is, the racing and the fading are fastest as a consequence of the low transaction costs. Taking the limit $\lambda \rightarrow 0$ (which, by change of units, is immediately seen to be equivalent to $T \rightarrow \infty$ ), L'Hôpital's rule yields for the overall surplus loss, the distressed trader's losses from predation, and the predator's gains:

$$
\begin{aligned}
\Delta V_{2} & \rightarrow \frac{\gamma}{6} \Delta x^{2}, \\
\Delta V_{d} & \rightarrow \frac{\gamma}{3} \Delta x^{2}, \\
V_{p} & \rightarrow \frac{\gamma}{6} \Delta x^{2} .
\end{aligned}
$$

In the limit, under market conditions that favor the most aggressive predation, the predator gains $\left(V_{p}\right)$, half of what the distressed trader loses $\left(\Delta V_{d}\right)$. This represents the lower bound for $\frac{V_{p}}{\Delta V_{d}}$.

\section{Cooperation and Liquidity}

To illustrate the incentives for cooperation in financial markets and the implications for market liquidity, we consider a dynamic game in which there are two strategic traders as well as a large number of long-term investors. Each player faces a common discount factor $\delta$ and the common asset price determinants $U_{t}, \gamma$, and $\lambda$. At the beginning of each stage, nature moves first and assigns a type to each of the traders. With probability $p_{i}, i=1,2$, each trader must liquidate a large position of size $\Delta x$. With probability $1-p_{i}$, each trader may act as a predator if their competitor needs liquidity. In each round, the traders have perfect information about each other's type. ${ }^{26}$

We assume that the distress probabilities $p_{1}$ and $p_{2}$ are mutually independent. In each time period one of the following four events occurs: neither of the two players is distressed, with probability $\left(1-p_{1}\right)\left(1-p_{2}\right)$; the second player is distressed but the first is not, with probability $\left(1-p_{1}\right) p_{2}$; the first player is distressed but the second is not, with probability $p_{1}\left(1-p_{2}\right)$; both players are distressed, with probability $p_{1} p_{2}$. The four probabilities sum to one. Cooperation is possible when either there exists one predator and one distressed trader

\footnotetext{
${ }^{26}$ In models of implicit collusion under imperfect information (Green and Porter (1984), Abreu, Pearce, and Stacchetti (1986)), players never deviate in equilibrium, but enter punishment phases because of exogenous price changes. Our game is one of complete information, where deviations cannot arise from such exogenous factors. This allows for a simpler model that captures the key issues of interest, namely how apparent liquidity arises from incentives to cooperate.
} 
(with probability $p_{1}+p_{2}-2 p_{1} p_{2}$ ), or when both players are distressed (with probability $p_{1} p_{2}$ ). If only one of the players is distressed and needs to liquidate a position, cooperation involves the other refraining from engaging in predatory trading. If both traders are distressed, cooperation involves both traders selling at a constant rate and refraining from racing each other to the market for their own gain.

Cooperation enables the players to quickly sell large blocks of shares for the price that would be obtained by selling them smoothly over time. That is, while cooperation is sustained, the distressed trader is allowed to walk down the demand curve, rather than having the information regarding the trading target quickly incorporated into the asset price ahead of most of their trading. In the sense that large blocks of shares can be moved for a better price, the market appears more liquid. Cooperation also avoids the volatility and potential instability from the large trading volume peaks that are associated with the racing and fading.

In what follows, we consider two versions of the model. As a building block, we develop a benchmark model in which the magnitude of the shock $\Delta x$ is constant. The two traders participate in an infinitely repeated game and the punishment strategy that they use is a grim-trigger strategy (Friedman (1971)). We determine which markets are prone to breakdowns in cooperation and analyze the effect of distress probabilities on the ability to support cooperation. We show that the ability to cooperate in markets has market structure implications.

Subsequently, we build upon the benchmark model and allow $\Delta x$ to be a stochastic random variable. The traders choose optimal strategies by taking expectations over $\Delta x$ and the distress probabilities that they face. In the equilibrium of this dynamic game, the traders implicitly agree not to punish each other for predating when the stakes are high. That is, they use a punishment strategy along the lines of Rotemberg and Saloner (1986). Episodically, cooperation breaks down leading to episodic illiquidity, which is short-lived. Finally, we consider the effect of multimarket contact and the contagion of illiquidity in the stochastic model.

\section{A. Fixed Liquidity Needs and Cooperation}

Let the two strategic traders play an infinitely repeated game in which the magnitude of the liquidity shock $\Delta x$ is constant. The punishment strategy considered is a trigger strategy in the spirit of Friedman (1971) and Dutta and Madhavan (1997). That is, for cooperation to occur in equilibrium for a given discount factor $\delta$, the expected value of a perpetuity of cooperation must exceed that of a one-time deviation plus a perpetuity of noncooperation. By the Folk theorem, a convex set of subgame-perfect Nash equilibria may exist in which intermediate levels of cooperation occur. For clarity of exposition, we focus on the extremal equilibrium that allows for the maximal cooperation.

The goal here is to predict when traders will abandon a cooperative effort, thereby leading to a reduction of the apparent liquidity in the market. We perform a comparative statics analysis by comparing the discount factor $\delta$ required 


\section{Table I}

\section{Expected Values from Strategic Trading}

Listed are the expected values that traders gain or lose when a sell-off occurs in the market. If only one trader is present, they sell a block of shares $\Delta x$ in the time interval $[0, T]$ and gain the value $V_{1}$. When a predator is present they will earn less and the loss they incur is $\Delta V_{d}=V_{1}-V_{d}$, where $V_{d}$ is the value they gain when they trade against the predator. The predator makes a profit of $V_{p}$ and the aggregate surplus to the predator and distressed trader is $V_{2}=V_{d}+V_{p}$. We show that the change in overall surplus, $\Delta V_{2}$, is always negative. The expressions in the right-hand column of the table are derived in the paper. $U_{0}$ is the expected value of future dividends, $\gamma$ measures the permanent impact of trading, and $\lambda$ measures the temporary impact of trading.

\begin{tabular}{lcl}
\hline $\begin{array}{l}\text { Surplus to distressed trader, } \\
\text { no predation }\end{array}$ & $V_{1}$ & $-U_{0} \Delta x-\left(\frac{1}{2}+\frac{1}{\frac{\gamma}{\lambda} T}\right) \gamma \Delta x^{2}$ \\
$\begin{array}{l}\text { Surplus to distressed trader } \\
\text { during predation }\end{array}$ & $V_{d}$ & $-U_{0} \Delta x-\frac{1}{6} \frac{5 e^{\frac{\gamma}{\lambda} T}+e^{\frac{2}{3} \frac{\gamma}{\lambda} T}+e^{\frac{1}{3} \frac{\gamma}{\lambda} T}-1}{e^{\frac{\gamma}{\lambda} T}-1} \gamma \Delta x^{2}$ \\
$\begin{array}{l}\text { Change in surplus to distressed } \\
\text { trader }\left(V_{1}-V_{d}\right)\end{array}$ & $\Delta V_{d}$ & $\left(\frac{1}{6} \cdot \frac{2 e^{\frac{\gamma}{\lambda} T}+e^{\frac{2}{3} \frac{\gamma}{\lambda} T}+e^{\frac{1}{3} \frac{\gamma}{\lambda} T}+2}{e^{\frac{\gamma}{\lambda} T}-1}-\frac{1}{\frac{\gamma}{\lambda} T}\right) \gamma \Delta x^{2}$ \\
$\begin{array}{l}\text { Surplus to predator } \\
\begin{array}{l}\text { Total surplus to predator } \\
\text { and distressed trader }\end{array}\end{array}$ & $V_{p}$ & $\frac{1}{6} \cdot \frac{e^{\frac{2}{3} \frac{\gamma}{\lambda} T}-1}{e^{\frac{2}{3} \frac{\gamma}{\lambda} T}+e^{\frac{1}{3} \frac{\gamma}{\lambda} T}+1} \gamma \Delta x^{2}$ \\
$\begin{array}{c}\text { Change in overall surplus } \\
\text { with predatory trading }\end{array}$ & $V_{2}$ & $-U_{0} \Delta x-\frac{1}{3} \cdot \frac{2 e^{\frac{1}{3} \frac{\gamma}{\lambda} T}-1}{e^{\frac{1}{3} \frac{\gamma}{\lambda} T}-1} \gamma \Delta x^{2}$ \\
& $\Delta V_{2}$ & $\left(\frac{1}{6} \cdot \frac{e^{\frac{1}{3} \frac{\gamma}{\lambda} T}+1}{e^{\frac{1}{3} \frac{\gamma}{\lambda} T}-1}-\frac{1}{\frac{\gamma}{\lambda} T}\right) \gamma \Delta x^{2}$
\end{tabular}

for cooperation to be possible under different scenarios of the other problem parameters. Given any particular punishment scheme, such as the more complicated penal codes in Abreu (1988), a critical $\delta$ can be derived. For this analysis, we do not allow the traders to change punishment schemes to achieve cooperation. We focus on trigger strategies because they lead to the same economic results while maintaining clarity of the model.

The following result describes the extremal equilibrium of our repeated game with fixed liquidity needs $(\Delta x)$ in each period, using a trigger strategy. Table I presents the expected values derived in Section I.

Result 4 (repeated game with two symmetric traders): Define the expected values as in Section I. When a trigger strategy (punishment strategy) is used, the discount factor required to support collusion is

$$
\delta \geq \delta_{\min }=\max \left\{\delta_{1}, \delta_{2}\right\},
$$

where the $\delta_{i}$ are the lowest discount factors for which each trader does not have an incentive to predate given the opportunity to do so (and given that the same is true for the other trader), which are 


$$
\begin{aligned}
& \delta_{1}=\frac{V_{p}}{p_{1}\left(1-p_{2}\right) \Delta V_{d}+2 p_{1} p_{2} \Delta V_{2}+\left(1-\left(1-p_{1}\right) p_{2}\right) V_{p}}, \\
& \delta_{2}=\frac{V_{p}}{\left(1-p_{1}\right) p_{2} \Delta V_{d}+2 p_{1} p_{2} \Delta V_{2}+\left(1-p_{1}\left(1-p_{2}\right)\right) V_{p}} .
\end{aligned}
$$

The $\delta_{1}$ and $\delta_{2}$ bounds on the discount factor (and therefore $\delta_{\min }$ ) are monotonically increasing in $\lambda$ and $T$, and monotonically decreasing in $\gamma$.

Result 4 predicts that cooperation is more likely for assets with a higher permanent price impact of trading, since the minimum discount factor $\delta$ for which cooperation can be supported is monotonically decreasing in $\gamma$. In Section I.C, we show that the surplus loss to predatory trading is monotonically increasing in $\gamma$. Since with higher $\gamma$ there exists a higher Pareto improvement available, it becomes more desirable for the traders to maintain cooperation. In contrast, in markets with a high temporary impact of trading $\lambda$, we would expect a lower level of cooperation. Since the Pareto improvement available monotonically decreases in $\lambda$, the level of cooperation should be lower for these markets.

Given Result 4, we can focus on the ratio $\frac{\gamma}{\lambda}$ to predict whether there will exist more or less aggressive cooperation. For large $\frac{\gamma}{\lambda}$, we expect cooperation to dominate predation. For small $\frac{\gamma}{\lambda}$, we expect predation to be more prevalent. The ratio $\frac{\gamma}{\lambda}$ impacts the liquidity available in asset markets. In Section II.B, we show that markets with a large $\frac{\gamma}{\lambda}$ have relatively smooth liquidity most of the time, but have the most marked spikes of illiquidity. These results arise because the monotonicities in Result 4 hold in the stochastic model.

Given Result 4, we can also analyze how $\delta$ relates to the probabilities of distress, that is, how the probabilities of each trader having future liquidity needs affects the traders' ability to cooperate. We will see that it is easier to support cooperation when the probabilities of distress are higher and more symmetric.

Consider the example in Figure 4. The figure plots the minimum $\delta$ for which cooperation is feasible as a function of $\gamma$. The other parameters are $\Delta x=1, U_{0}=$ $10, T=10$, and $\lambda=1$. The base case is the solid line, in which $p_{1}=0.5$ and $p_{2}=0.5$. For all values of $\gamma$, the $\delta$ required for cooperation is less than one, which means that if the strategic traders are sufficiently patient they can cooperate in any market. Now consider the case in which $p_{1}=0.1$ and $p_{2}=0.1$. It is still possible to cooperate in any market, but the required $\delta$ is higher. As the probability of distress decreases, a player that is not distressed and has the opportunity to predate will likely have to wait longer until the next event in which they might benefit from cooperation from the other player. For the value of future benefits from cooperation to be sufficient for the player not to have an incentive to predate, the future needs to be less discounted (i.e., higher $\delta$ ). Finally, consider the case where $p_{1}=0.5$ and $p_{2}=0.3$. In this case, it is not possible for the traders to cooperate in markets with low $\gamma$. Since we must have $\delta \in[0,1]$, we can find a bound on $\gamma / \lambda$ below which cooperation should never be observed. Symmetry in distress probabilities between the traders is an 


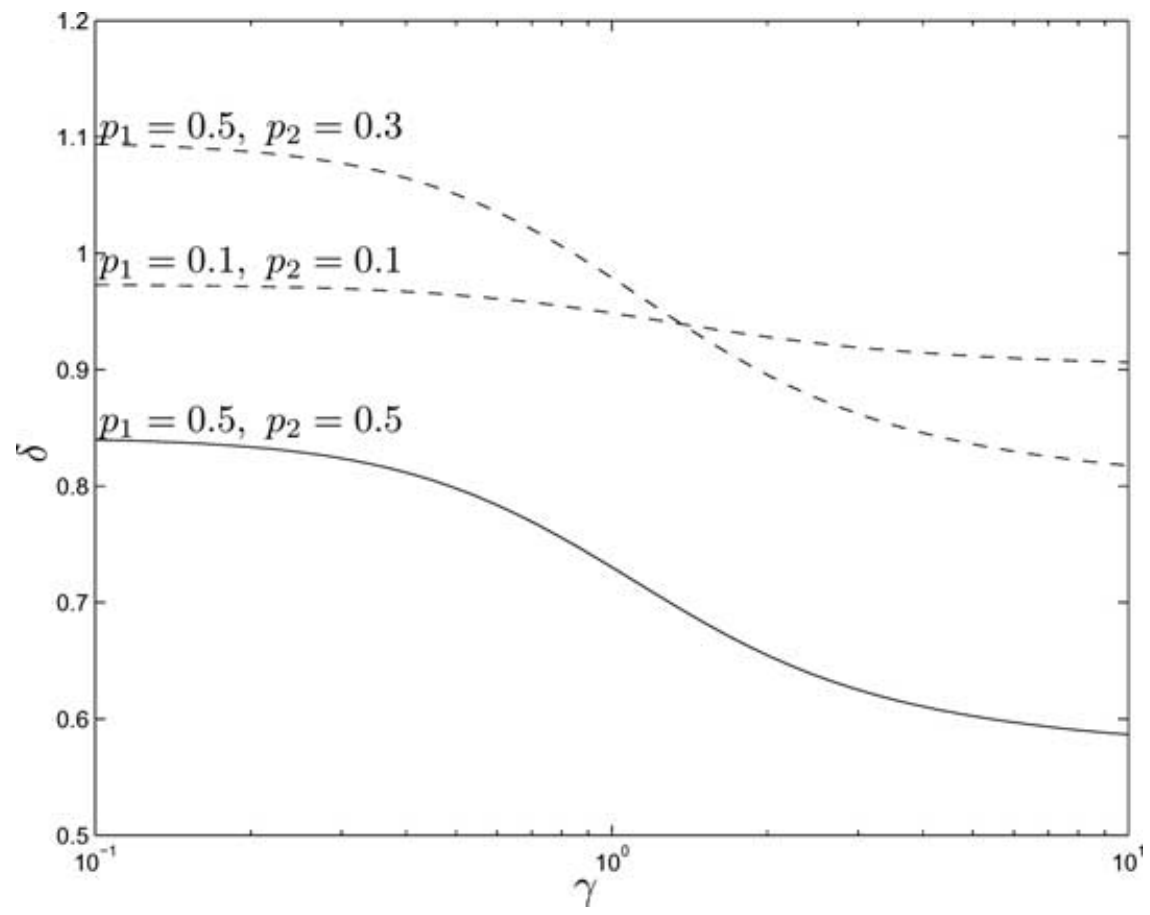

Figure 4. Minimum $\delta$ for which cooperation is feasible as a function of $\gamma$, with logarithmic scale on the $\gamma$-axis. Three different cases of the distress probabilities of each trader are plotted (the probabilities are assumed independent). It is always possible to support cooperation when $p_{1}=p_{2}=0.5$ and when $p_{1}=p_{2}=0.1$, but it is harder when the distress probabilities are lower. When the permanent price impact parameter $\gamma$ is low it is impossible support cooperation when the distress probabilities are asymmetric $\left(p_{1}=0.5\right.$ and $\left.p_{2}=0.3\right)$. The other parameters are $\Delta x=1, U_{0}=10, T=10$, and $\lambda=1$.

important factor for cooperation. In markets with a low $\gamma / \lambda$, even a small degree of asymmetry will be enough to cause the traders to abandon a cooperative relationship.

These conclusions are supported analytically as follows. Without loss of generality, assume $\delta_{1}=\delta_{\min }$ (that is, $p_{1}<p_{2}$ ). Equation (19) can be rewritten as

$$
\delta_{1}=\frac{V_{p}}{p_{2}\left[p_{1} \Delta V_{2}-V_{p}\right]+p_{1} \Delta V_{d}+V_{p}} .
$$

From equation (20) we can see that $\delta_{1}$ is monotonically increasing in $p_{2}$ (since we can establish that $V_{p} \geq \Delta V_{2}$ from $V_{p} / \Delta V_{d}>1 / 2$ in Result 3 and $\Delta V_{d}=\Delta V_{2}+$ $V_{p}$ ). A larger probability of distress for the larger trader makes cooperation by the smaller trader more difficult (cooperation is possible only under a narrower range of market conditions). We can also rewrite equation (19) as 


$$
\delta_{1}=\frac{V_{p}}{p_{1}\left[\left(1-p_{2}\right) \Delta V_{d}+2 p_{2} \Delta V_{2}+p_{2} V_{p}\right]+\left(1-p_{2}\right) V_{p}},
$$

from which we see that $\delta_{1}$ is monotonically decreasing in $p_{1}$. A larger probability of distress for the smaller trader makes cooperation easier (possible under a wider range of market conditions).

Using Result 4 it is possible to place bounds on how symmetric the distress probabilities must be in order to support cooperation. Consider, for example, the case in which the traders are infinitely patient $(\delta=1)$ and, without loss of generality, $p_{1}<p_{2}$. To support cooperation it must be that

$$
p_{1} \geq p_{2} \frac{V_{p}}{\Delta V_{d}}-p_{1} p_{2} \frac{\Delta V_{2}}{\Delta V_{d}} .
$$

Evaluating equation (22) under extreme market conditions $(\gamma / \lambda \rightarrow 0$ and $\gamma / \lambda \rightarrow \infty)$ allows us to derive the relative values of the distress probabilities for which cooperation is possible. ${ }^{27}$ Taking the limits above, equation (22) becomes

$$
p_{1} \geq \frac{4}{5} p_{2}-\frac{1}{5} p_{1} p_{2} \quad \text { and } \quad p_{1} \geq \frac{1}{2} p_{2}-\frac{1}{2} p_{1} p_{2} .
$$

Another useful relation follows from the case in which distress events are infrequent $\left(p_{1}, p_{2} \ll 1\right)$. The probably of both players being simultaneously distressed is then negligible. The size of the smaller player relative to larger player is then bounded by

$$
\frac{p_{1}}{p_{2}} \geq \frac{V_{p}}{\Delta V_{d}}
$$

In the limit cases above $(\gamma / \lambda \rightarrow 0$ and $\gamma / \lambda \rightarrow \infty)$, this is

$$
\frac{p_{1}}{p_{2}} \geq \frac{4}{5} \text { and } \quad \frac{p_{1}}{p_{2}} \geq \frac{1}{2} \text {. }
$$

As we discuss above, the traders' distress probabilities need to be sufficiently symmetric for cooperation to be possible. Equation (25) provides some insight on the required level of symmetry.

The requirement for symmetry in distress probabilities has implications for market structure. Strategic traders often trade on behalf of external clients who use these markets. For example, proprietary trading desks trade for both their clients and on their own account. If the probability of needing to trade large blocks in a short time period is linked to the market share of external clients that a trader serves, the model predicts that active traders may have an incentive to share the market with their competition. If a duopoly exists (when a monopoly is not possible), it may be to the benefit of a large trader to allow a smaller trader to grow in size so that a Pareto superior outcome for the

\footnotetext{
${ }^{27}$ The limits $\frac{\gamma}{\lambda} \rightarrow 0$ and $\frac{\gamma}{\lambda} \rightarrow \infty$ are defined as follows. The limit $\frac{\gamma}{\lambda} \rightarrow 0$ is achieved by letting $\gamma \rightarrow 0, \lambda \rightarrow \infty$, or both. The limit $\frac{\gamma}{\lambda} \rightarrow \infty$ is achieved analogously.
} 
strategic traders can be achieved. This incentive to share the market will need to be weighed against the benefits of having more business for oneself. However, should the surplus from cooperation be lost, the value of being an insider will be eroded, and with it the ability to extract rents from external clients.

Consider the following scenario in which there exist two strategic traders and a representative outsider who seeks to trade a block of an asset. The strategic traders have two alternatives when an outsider needs to trade. They may initiate a predatory strategy, racing and fading the external player to the market and earning a profit by affecting the price of the asset. Alternatively, the outsider may become a client of the traders so that the traders may exact rents for use of their services (these rents may arise in the form of a bid-ask spread). The fact that there exists a cooperative outcome in this market between the insiders provides a means by which a relatively stable, albeit widened, bid-ask spread may exist, and we do not necessarily observe price volatility when a nonmember needs liquidity. The amount of the surplus available between the traders and the client is $\Delta V_{d}$, since the outsider is indifferent between receiving $V_{d}$, and paying $\Delta V_{d}$ in order to receive $V_{1}$ when using the services of the strategic traders. ${ }^{28}$

The external client uses each trader with probabilities $p_{1}$ and $p_{2}$. Equation (22) implies that the relative market shares of the traders should be reasonably symmetric to support cooperation. Consider the example in Figure 5 in which the minimum $\delta$ necessary to support cooperation is plotted as a function of $p_{1}$. Two ranges of scenarios are illustrated: $p_{1}=p_{2}$ (bold line) and $p_{1}+p_{2}=0.5$ (dotted line). When the traders' distress probabilities (market shares) are symmetric, cooperation is always possible as long as traders are sufficiently patient. However, when the market shares are asymmetric and $p_{1}<0.18$ (36\% market share) or $p_{1}>0.32$ (64\% market share), cooperation is not possible. If one trader has a market share larger than $64 \%$, they may find it in their own interest to allow their opponent to gain market share so that the ongoing Pareto-superior relationship may continue. This may provide an explanation for the observation in practice of deviations in the bid-ask spread without resulting in price wars.

Figure 6 illustrates, for three different values of $\delta$, the $\left(p_{1}, p_{2}\right)$ pairs that can support cooperation. The values of $\delta$ that are plotted are $1,0.9$, and 0.8 . The other parameters are $\Delta x=1, U_{0}=10, T=10, \gamma=1$, and $\lambda=1$. The shaded region corresponds to the $\left(p_{1}, p_{2}\right)$ pairs for which cooperation is possible if both traders use a discount factor $\delta=0.8$. Note that the boundaries of the sets are not straight lines due to the bilinear terms $p_{1} p_{2}$, but are nearly so for small values of $p_{1}$ and $p_{2}$. For $\delta=1$, and for small probabilities, the set boundaries go to the origin with slope $V_{p} / \Delta V_{d}$ and $\Delta V_{d} / V_{p}$. As the sum of the two probabilities becomes smaller, traders are required to be of more similar sizes for a cooperative outcome to be feasible. Note that the bold and dashed lines correspond to

\footnotetext{
${ }^{28}$ To determine the division of this surplus between the insiders and the external player, it is possible to use a generalized Nash bargaining solution in which the insiders receive fraction $\tau$ of the surplus and the client receives fraction $1-\tau$. The example that we consider (Figure 5) is equivalent to assuming $\tau=1$. This is without any significant loss of generality, since relaxing this assumption leads to the same comparative statics.
} 


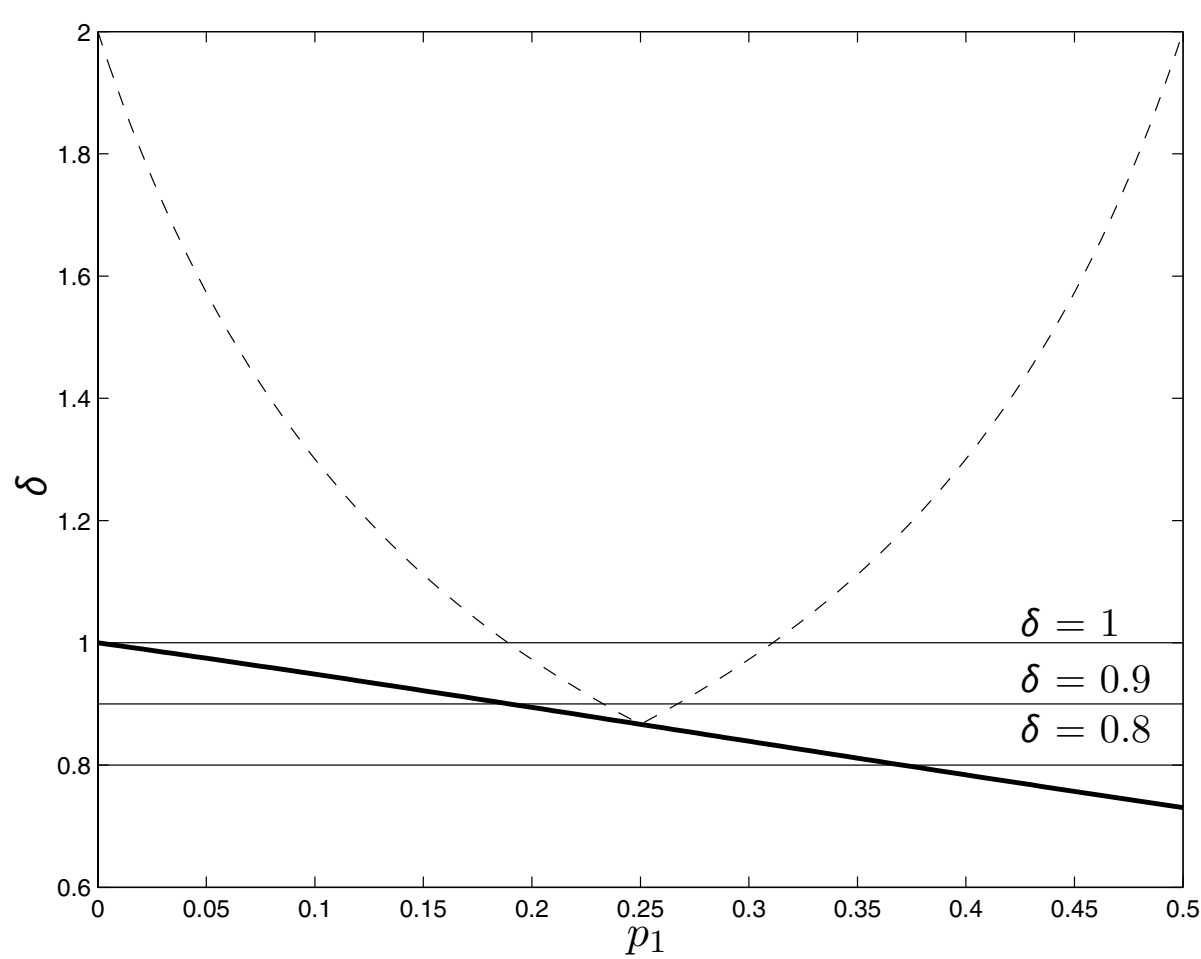

Figure 5. Minimum $\delta$ as a function of distress probabilities, for the cases $p_{1}=p_{2}$ (bold) and $p_{2}=0.5-p_{1}$ (dashed). Parameters are $\Delta x=1, U_{0}=10, T=10, \gamma=1$, and $\lambda=1$. The horizontal reference lines correspond to the iso- $\delta$ lines in Figure 6.

the cases plotted in Figure 5. Considering cases with smaller overall frequency of events $p_{1}+p_{2}$ corresponds to moving the dashed line to the lower-left. Figures 5 and 6 illustrate the result that market shares need to be sufficiently symmetric for cooperation to be possible.

In the next section, we consider these relationships when the liquidity event $(\Delta x)$ is stochastic across time. We also discuss the effect of multimarket contact and contagion of illiquidity.

\section{B. Episodic Illiquidity and Contagion}

Shocks of Random Magnitude. In Section II.A, we evaluate the requirements for cooperation given that $\Delta x$ is a fixed amount of the asset. In that formulation, if cooperation is possible (based on the market parameters and $\delta$ ), the traders never deviate. To characterize episodic illiquidity, $\Delta x$ is better modeled as a random variable. In the event of a large $\Delta x$, it is more profitable for the traders to deviate for a one-time gain. However, instead of initiating a grim-trigger strategy, there are more profitable strategies available to the cartel. 


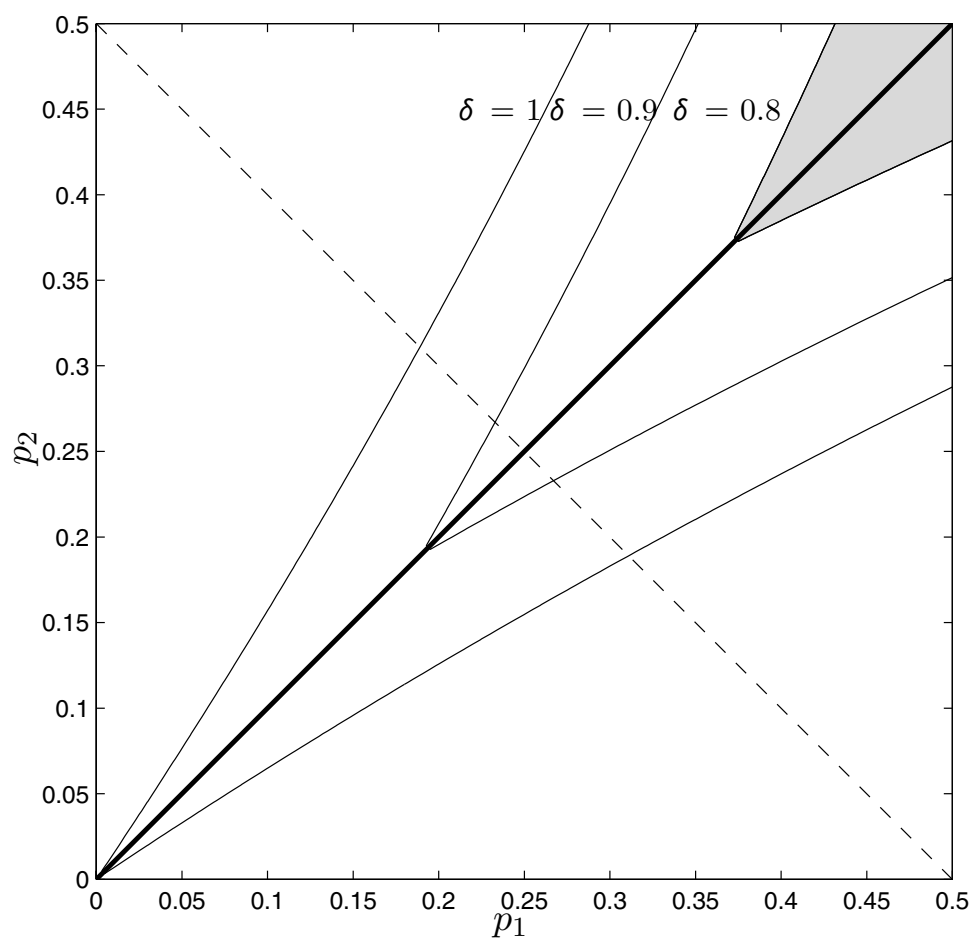

Figure 6. Values of $p_{1}$ and $p_{2}$ for which cooperation can be sustained, given $\delta$ (plotted for the cases $\delta=\mathbf{1}, \delta=\mathbf{0 . 9}$, and $\delta=\mathbf{0 . 8}$ ). For instance, for $\delta=0.8$ the cooperative equilibrium exists if $p_{1}$ and $p_{2}$ fall in the shaded region. The bold and dashed lines indicate the values of $p_{1}$ and $p_{2}$ that are plotted in Figure 5.

The large traders implicitly agree to restrain from predating when the magnitude of the shock is below some threshold $\Delta x^{*}$ and, conversely, not to punish other players in future periods for predating when the shock is above that threshold. That is, when a player has a trading requirement that exceeds $\Delta x^{*}$, the other player will predate, but cooperation is resumed in subsequent periods. This equilibrium behavior results in episodically increased volatility. ${ }^{29}$

Another way to describe this equilibrium is that each trader agrees to restrain from predating on the other, but only as long as they behave responsibly in their risk management. This creates a natural restriction on the exposure that each trader can take without a disproportionate increase in the risk of their portfolio.

The value of $\Delta x^{*}$ that is optimal for the cartel (in the sense of leading to the highest expected value for its members) can be computed for any distribution

\footnotetext{
${ }^{29}$ Episodic illiquidity also occurs during extreme financial distress. During extreme distress, a member of the oligopoly becomes a finite concern. Because the horizon of this game is finite, the players work out their strategy profiles by backward induction and cooperation disappears.
} 
of the trading requirement for each trader. In general, $\Delta x^{*}$ can only be characterized implicitly.

RESUlT 5 (shocks of random magnitude): Consider trading requirements for each of two traders, which are shocks of random magnitude $\Delta x$ identically and independently distributed according to the density $f(y)$, which we assume to be

(i) symmetric, $f(y)=f(-y)$,

(ii) with unbounded support, $f(y)>0, \forall y \in \mathbb{R}$,

(iii) and with finite variance, $\int_{-\infty}^{\infty} y^{2} f(y) d y<\infty$.

A strategy with episodic predation with threshold $\Delta x^{*}$ is feasible for any $\Delta x^{*}$ that satisfies

$$
2 C \int_{0}^{\Delta x^{*}} y^{2} f(y) d y \geq K\left(\Delta x^{*}\right)^{2} .
$$

The supremum of $\Delta x^{*}$ such that the inequality is satisfied exists, and we designate it by $\overline{\Delta x}$. The following strategy profile constitutes a subgame-perfect Nash equilibrium. At time $t=0$, predate if $|\Delta x|>\Delta x^{*}$, and otherwise cooperate. At time $t \neq 0$,

1. If the history of play $h^{t-1}$ is such that for every period in which $|\Delta x|<\Delta x^{*}$ there was no predation, then

(a) if $|\Delta x|>\Delta x^{*}$, predate this period.

(b) if $|\Delta x|<\Delta x^{*}$, cooperate.

2. If $h^{t-1}$ is such that for $|\Delta x|<\Delta x^{*}$, there was predation, then predate.

The constants above are

$$
C=\frac{\delta}{1-\delta}\left[p_{1}\left(1-p_{2}\right) K_{d}+2 p_{1} p_{2} K_{2}-\left(1-p_{1}\right) p_{2} K\right]
$$

and $K=V_{p} / \Delta x^{2}$, with $K_{d}=\Delta V_{d} / \Delta x^{2}$ and $K_{2}=\Delta V_{2} / \Delta x^{2}$ (that is, $K, K_{d}$, and $K_{2}$ are the factors multiplying $\Delta x^{2}$ in the expected values $V_{p}, \Delta V_{d}$, and $\Delta V_{2}$; note that $K$ in equation (26) is the factor in the expected gain to the predator).

In what follows, the parameter $C / K$ can be interpreted as representing the benefits relative to the costs of cooperation. For each of the players $i$, the numerator $C$ is increasing in the discount rate $(\delta)$, increasing in $p_{i}$, and decreasing in $p_{j \neq i}$. The ratio $C / K$ is also increasing in $K_{d}$ (the welfare loss if player $i$ is distressed and player $p_{j \neq i}$ is not), increasing in $K_{1}$ (the total welfare loss if both are distressed and do not cooperate), and decreasing in $K$ (the profit from predation). Thus, $C / K$ is a measure of the relative strengths of the incentives to cooperate and predate.

To gain intuition about how the parameter $C / K$ affects market liquidity, let the shock $\Delta x$ be normally distributed. In Figure 7, the left-hand side of the inequality in equation (26) is plotted with the solid line and the right-hand side 


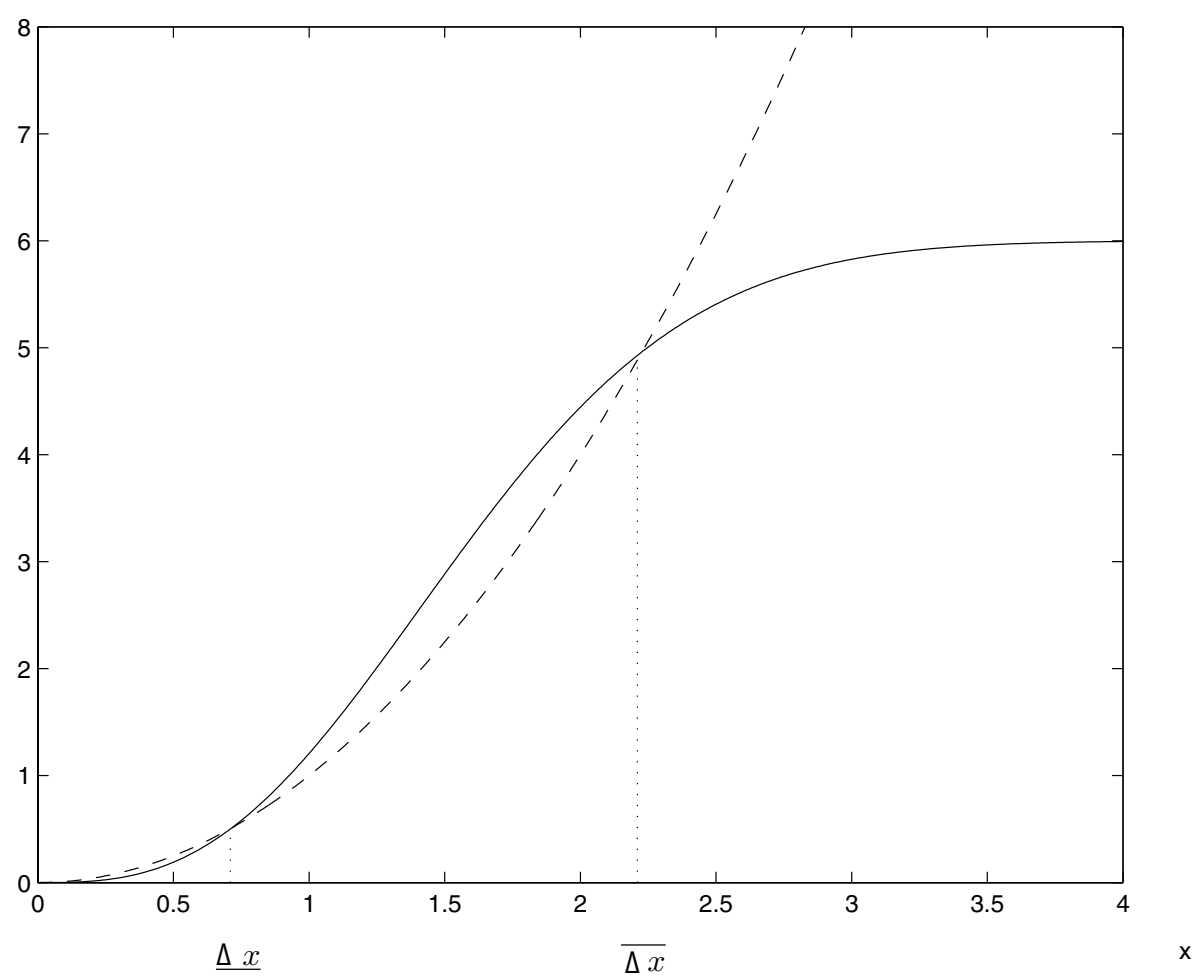

Figure 7. Left- (solid) and right-hand side (dashed) of equation (26). The curves intersect at zero, $\underline{\Delta x}$, and $\overline{\Delta x}$.

is plotted with the dashed line. For $\Delta x^{*}$ in an interval $[\underline{\Delta x}, \overline{\Delta x}]$, it is possible to sustain the subgame-perfect Nash equilibrium. For $\Delta x^{*}>\overline{\Delta x}$, the value gained for deviation is too high and cooperation cannot be maintained. The supremum $\overline{\Delta x}$ defines the most profitable strategy for the cartel. (Note that $\overline{\Delta x}$ might be zero, in which case traders never cooperate.)

The nature of the solutions is essentially independent of the scale parameter of the distribution. Consider a family of distributions $f_{a}(y)=a f(a y)$. If we determine solutions in terms of $\Delta x^{*} / a$, the set of feasible thresholds is independent of the asset parameters. The inequality in equation (26) is equivalent to

$$
2 \frac{C}{K} \int_{0}^{\frac{\Delta x^{*}}{a}} y^{2} f_{a}(y) d y \geq\left(\frac{\Delta x^{*}}{a}\right)^{2},
$$

so that, after the corresponding scaling, the solutions to the inequality are constant with scaling of the distribution. For example, if we consider the zeromean normal distribution 


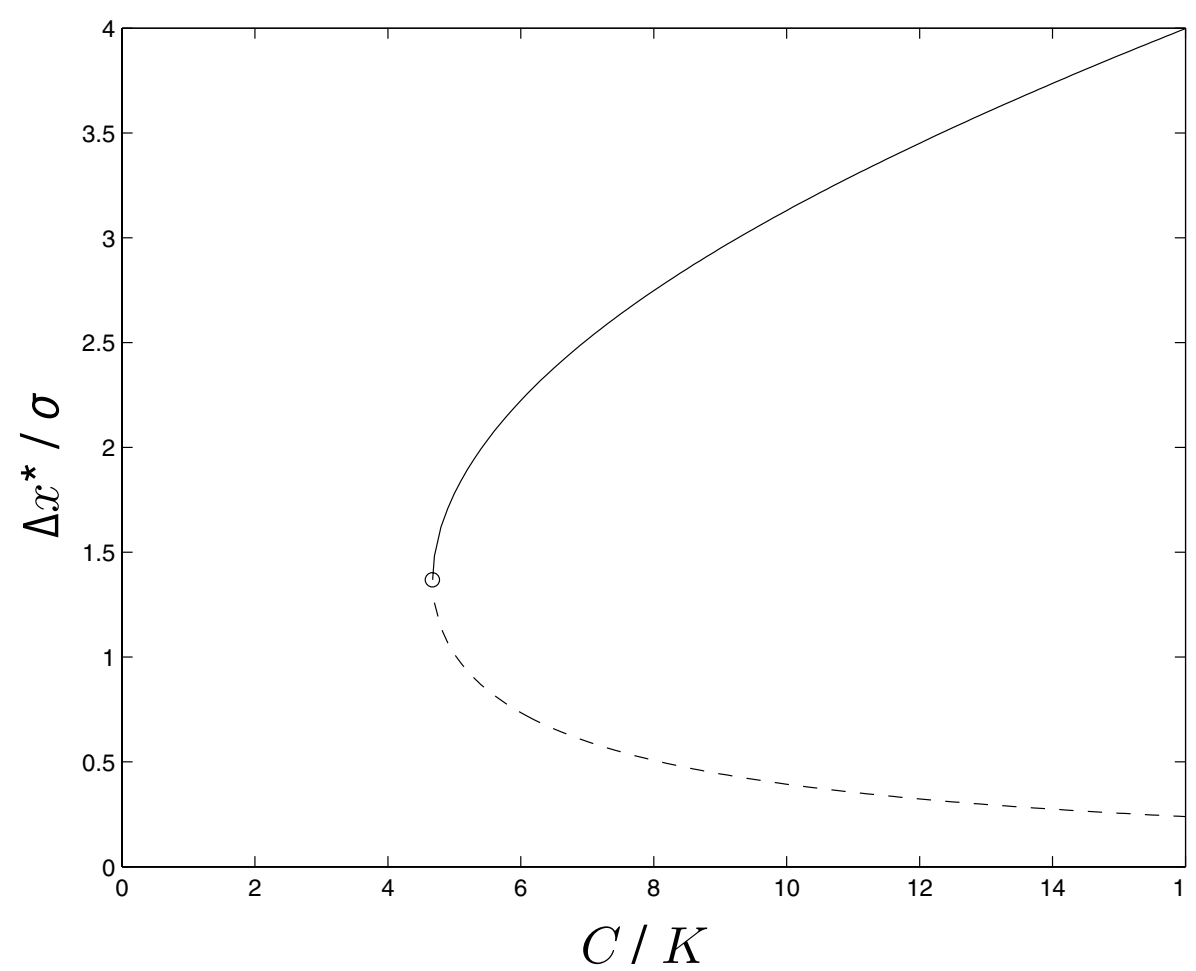

Figure 8. Normally distributed shocks, upper and lower bounds for $\Delta x^{*} / \sigma$ as a function of the asset parameters. The ratio $C / K$ measures the benefits from cooperation relative to the costs of cooperating in financial markets. The open circle at $C / K=4.6729$ is the minimum $C / K$ for which there exists a nonzero $\overline{\Delta x}$, that is, for which an episodic predation strategy is feasible.

$$
f(y)=\frac{1}{\sigma \sqrt{2 \pi}} e^{-\frac{1}{2} \frac{y^{2}}{\sigma^{2}}},
$$

we can rewrite the inequality as

$$
\frac{K}{C}\left(\frac{\Delta x^{*}}{\sigma}\right)^{2} \leq 2 \int_{0}^{\frac{\Delta x^{*}}{\sigma}} y^{2} \frac{1}{\sqrt{2 \pi}} e^{-\frac{1}{2} y^{2}} d y .
$$

Therefore, we can parameterize any such problem using only $C / K$ and $\Delta x^{*} / \sigma$.

Figure 8 plots $\underline{\Delta x} / \sigma$ and $\overline{\Delta x} / \sigma$ as a function of $C / K$. The solid line represents $\overline{\Delta x} / \sigma$ and the dotted line represents $\Delta x / \sigma$. For any value of $C / K$, the vertical segment between the lines is the set of $\Delta x^{*}$ (in standard deviations) such that cooperation is possible. Note that there exists a critical value for $C / K$ (represented by the small circle), below which it is impossible to support cooperation. Any threshold we might consider would always be too high, in that the immediate gains from deviation provide an incentive to deviate, and too small, in that the expected future rewards from cooperation during those shocks that do fall below the threshold do not provide sufficient incentive to cooperate. 
We can now determine the minimum or critical $C / K$ for which there is a nonzero $\overline{\Delta x}$, that is, for which an episodic predation strategy is feasible. Consider again the normal distribution and the inequality in equation (30). Taking the derivative of the difference between the two sides of the inequality with respect to $\Delta x^{*}$ and equating it to zero leads to

$$
\frac{K}{C}=\frac{1}{\sqrt{2 \pi}} \frac{\Delta x^{*}}{\sigma} e^{-\frac{1}{2}\left(\frac{\Delta x^{*}}{\sigma}\right)^{2}} .
$$

This characterizes the points in Figure 7 at which the solid and dashed lines have the same derivative. Using this at the supremum (i.e., with equality holding in equation (30)), we obtain the case in which the lines touch at a single point rather that having two intersections (other than zero). After a change of variable in the integral, we obtain

$$
\int_{0}^{\frac{\Delta x^{*}}{\sigma}} y^{2} \frac{1}{2 \pi} e^{-\frac{1}{2} y^{2}} d y=\frac{1}{2}\left(\frac{\Delta x^{*}}{\sigma}\right)^{3} \frac{1}{\sqrt{2 \pi}} e^{-\frac{1}{2}\left(\frac{\Delta x^{*}}{\sigma}\right)^{2}},
$$

which is straightforward to solve numerically for $\Delta x^{*} / \sigma$. Since $C / K$ only depends on $\Delta x^{*}$ through $\Delta x^{*} / \sigma$, the minimum $C / K$ ratio for which there is a feasible strategy of the episodic predation type does not depend on the scale parameter of the distribution. For the normal distribution, the minimum $C / K$ for which there is a nonzero $\overline{\Delta x}$, that is, for which an episodic predation strategy is feasible, is $C / K=4.6729$ for any $\sigma$. This imposes restrictions on the parameters. For assets with parameters such that $C / K$ is less than this critical value, no episodic predation strategy is feasible. The threshold associated with this $C / K$ ratio is $\Delta x=\overline{\Delta x}=1.3688 \sigma$.

In Section II.A, we showed that the ability for traders to cooperate when the liquidity needs are fixed is increasing in $\frac{\gamma}{\lambda}$. By Result 3, it is easy to show that $C / K$ is also monotonically increasing in $\gamma$ and monotonically decreasing in $\lambda$. This implies that markets with high $\frac{\gamma}{\lambda}$ will have a higher $C / K$. Based on this observation, the following predictions are natural. For assets with a high level of asymmetric information (high $\gamma$ ) that are widely traded in large volume (low $\lambda$ ), we would expect liquidity to be stable most of the time (apparent liquidity), but to disappear episodically. An example of this type of security would be a growth stock (perhaps a tech stock) with diffuse ownership. In contrast, a low $\frac{\gamma}{\lambda}$ asset may have liquidity levels that fluctuate more regularly, but that do not exhibit marked illiquidity in an episodic way. An example of a low $\frac{\gamma}{\lambda}$ asset would be a thinly traded AA-rated corporate bond. To our knowledge, securities have not been previously grouped into these categories and studied for their permanent and temporary illiquidity, as is necessary to test the empirical implications of our model.

Contagion across Markets. Suppose that the members of the oligopoly can cooperate in more than one market. As an example, consider institutional traders who dominate mortgage markets and are also strategic traders in other fixed income markets. If a liquidity event is large enough to disturb cooperation in 


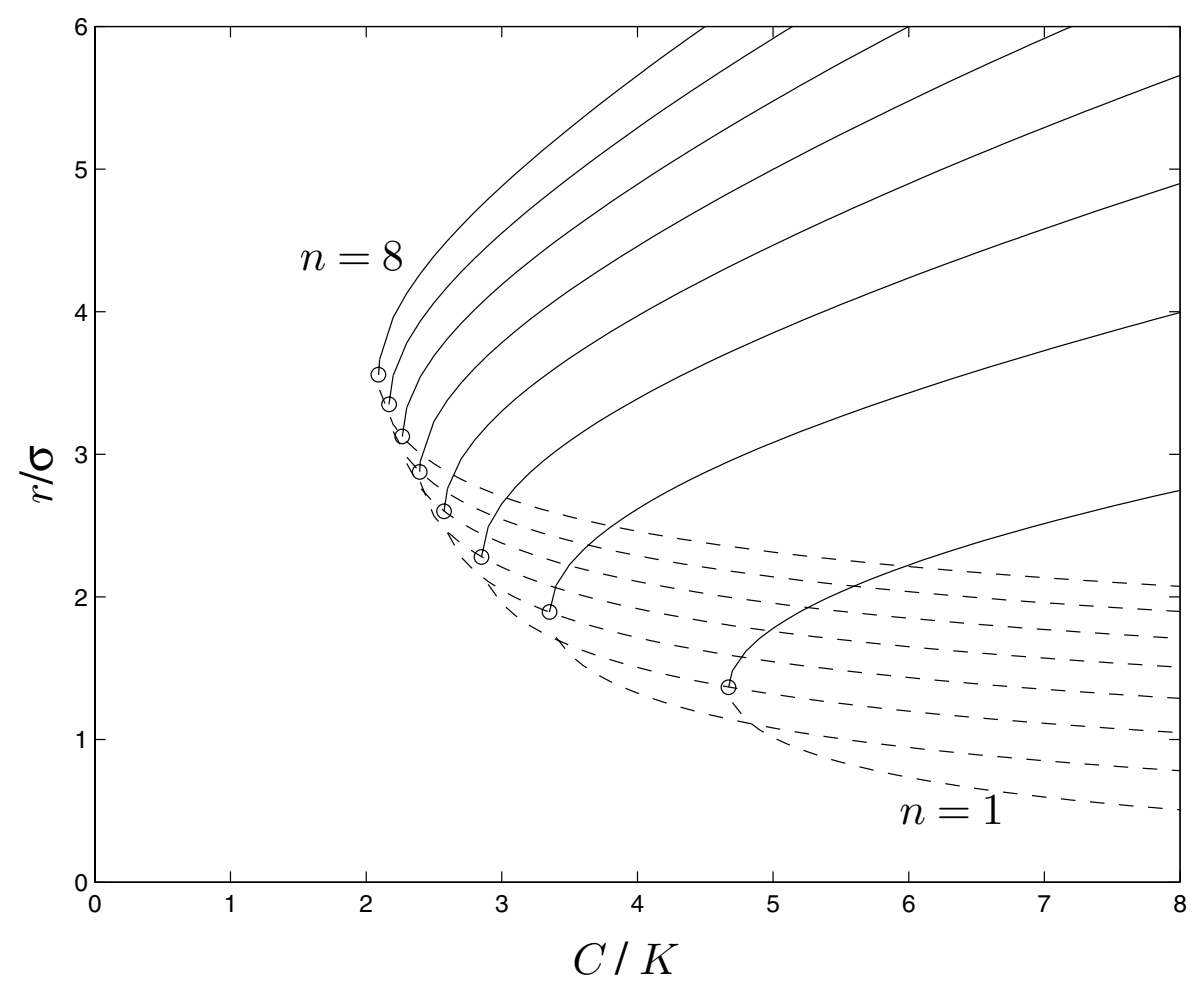

Figure 9. Lowest and highest $r$ such that the strategy (episodic predation with contagion over $n$ assets, $n=1,2, \ldots, 8$ ) is an equilibrium, plotted as a function of the asset parameters, common to all assets. The shocks are independent and normally distributed conditional on shocks being either all positive or all negative. The ratio $C / K$ measures the benefits from cooperation relative to the costs of cooperating in financial markets. The open circle for each curve is the minimum $C / K$ for which there exists a non-zero $\overline{\Delta x}$, that is, for which an episodic predation strategy is feasible. Note that the minimum $C / K$ decreases as the number of markets $n$ increases.

one market, it may also affect cooperation in the others. Bernheim and Whinston (1990) find that if markets are not identical, multimarket contact supports cooperation. In our case, and since assets are not perfectly correlated, multimarket contact makes it easier to maintain cooperation. In this section, we discuss the effects of multimarket contact on episodic illiquidity across markets.

Consider traders that participate in $n$ markets, where the trading requirement in each market is a stochastic random variable. We define a liquidity event to be such that all trading targets for each of the $n$ assets have the same sign (i.e., liquidity shocks occur in the same direction in all markets). ${ }^{30}$ The trading

${ }^{30}$ Implicitly, we are assuming that there is a common cause driving the liquidity event. That is, the liquidity needs for each trader are positively correlated across markets. We can generalize the model to account for arbitrary correlation. However, it seems less plausible that a trader would simultaneously have both large positive and large negative liquidity needs. 
Table II

Multimarket Contact across $n$-Markets

The ratio $C / K$ measures the benefits from cooperation relative to the costs of cooperating in financial markets. For $n=1,2, \ldots, 20$, we calculate the minimum values of $C / K$ for which cooperation is possible over multiple markets. As the number of markets increases ( $n$ increases), the relative benefit needed to support cooperation decreases.

\begin{tabular}{rrrrrrrr}
\hline$n$ & $C / K$ & $n$ & $C / K$ & $n$ & $C / K$ & $n$ & $C / K$ \\
\hline 1 & 4.6729 & 6 & 2.2664 & 11 & 1.9299 & 16 & 1.7740 \\
2 & 3.3509 & 7 & 2.1687 & 12 & 1.8907 & 17 & 1.7517 \\
3 & 2.8507 & 8 & 2.0913 & 13 & 1.8563 & 18 & 1.7314 \\
4 & 2.5747 & 9 & 2.0280 & 14 & 1.8259 & 19 & 1.7127 \\
5 & 2.3950 & 10 & 1.9751 & 15 & 1.7986 & 20 & 1.6954 \\
\hline
\end{tabular}

targets are modeled as jointly normal and conditionally independent given that they are either all positive or all negative. We also use the simplifying assumption that trading targets in all the assets have the same variance. The density in the positive orthant ( $y$ such that $y_{i} \geq 0$, for all $i$ ) and in the negative orthants $\left(y\right.$ such that $y_{i} \leq 0$, for all $i$ ) is

$$
f(y)=\frac{2^{n-1}}{\sigma^{n}(2 \pi)^{n / 2}} e^{-\frac{y^{T} y}{\sigma^{2}}},
$$

and zero elsewhere.

The shape of the optimal region for cooperation is spherical. This is the region in which the incentive to predate, which is proportional to $\sum_{i=1}^{n} \Delta x_{i}^{2}$, is constant. The inequality for $n$ assets involves an $n$-dimensional integral, which using the radial symmetry of the normal distribution, can be written as

$$
2 C \int_{0}^{r} S_{n} y^{n+1} f(y) d y \geq K r^{2},
$$

where $r$ is the radius of the cooperation region and

$$
S_{n}=\frac{1}{2^{n}} \frac{2 \pi^{\frac{n}{2}}}{\Gamma\left(\frac{n}{2}\right)}
$$

is the area of the intersection of the $n$-dimensional sphere with unit radius with the positive orthant. It can easily be verified that, as for the one-asset case, the nature of the solutions is essentially independent of the scale parameter of the distribution (say the standard deviation).

Figure 9 is the multimarket version of Figure 8 in that it plots $\Delta x$ and $\overline{\Delta x}$ for episodic predation over $n$ markets, $n=1,2, \ldots, 8$. The minimum value of $C / K$ that is required to support cooperation decreases as the number of markets increases. Adding markets can make cooperation possible where it would otherwise not be possible. This is because adding markets increases the relative value of cooperation, given that punishment is effected over all markets. 
(Consider, for instance, $C / K=4.0$. With these parameters, traders are unable to cooperate over one market, but are able to do so over two or more markets.) Also note that the supremum of $r$ increases with $n$. The probability that an episode of predation will occur is in fact seen to decrease with $n$. We expect episodes of predation to be more significant, since they now affect $n$ markets, but less frequent with contagion strategies. The minimum values of $C / K$ for cooperation over multiple markets, $n=1,2, \ldots, 20$, are listed in Table II.

\section{Conclusion}

We present a model where the breakdown of cooperation in financial markets leads to episodic illiquidity. This model is based on an equilibrium strategy in which traders cooperate most of the time through repeated interaction, providing apparent liquidity to one another. However, episodically this cooperation breaks down, especially when the stakes are high, leading to opportunism and loss of this apparent liquidity. Our model provides an explanation for why episodic liquidity breakdowns do not occur more often, and predicts that this apparent liquidity is more easily sustained in asset markets with a high permanent price impact of trading and a low temporary price impact of trading.

We solve a competitive trading game for strategic traders (predatory stage game), which is formulated as a continuous-time dynamic programming problem using an asset pricing equation that accounts for transaction costs from the price impact of trading. According to this model, traders race to market, selling quickly in the beginning of the period. Also while predators initially race distressed traders to market, they eventually fade them and buy back. The presence of predators in the market leads to a surplus loss to the strategic traders.

Cooperation in the market is modeled by embedding this predatory stage game in a dynamic game with infinite horizon. Cooperation allows for the trading of large blocks of the asset at more favorable prices, so that the surplus loss due to predatory trading can be avoided. This leads to predictions about the types of markets in which cooperation is more likely.

We believe that our model presents a plausible argument for the level of predation or cooperation in financial markets being a determinant of available liquidity, and a contributor to the episodic nature of illiquidity.

\section{Appendix A}

Proof of Result 1 (General solution): Given that each player's objective is linear in $U_{t}$ and that the strategies are open-loop, we can bring the expectation inside the integral (Fubini's theorem applies since the trading rate $Y_{t}^{i}$ is admissible) and consider the equivalent problem with a deterministic asset pricing equation. Replacing $U_{t}$ with a constant $u=U_{0}$,

$$
P_{t}=u+\gamma \sum_{j=1}^{n} X_{t}^{j}+\lambda \sum_{j=1}^{n} Y_{t}^{j}
$$


This is a standard deterministic control problem with objective concave in $X_{t}^{i}$ and $Y_{t}^{i}$ and constraint linear in the control $Y_{t}^{i}$. If the solutions to the necessary conditions are continuous, sufficiency follows.

With the multiplier function $Z_{t}^{i}$ associated with the constraint $d X_{t}^{i}=Y_{t}^{i} d t$, necessary optimality conditions for the problem faced by trader $i$ are (Basar and Olsder (1999))

$$
\begin{aligned}
& u+\gamma \sum_{j=1}^{n} X_{t}^{j}+\lambda \sum_{j=1}^{n} Y_{t}^{j}+\lambda Y_{t}^{i}+Z_{t}^{i}=0 \\
& d Z_{t}^{i}=-\gamma Y_{t}^{i} d t .
\end{aligned}
$$

The second equation ensures that the multiplier function is continuous (as required for sufficiency). Differentiating the first equation with respect to $t$ and substituting the second,

$$
\gamma \sum_{j=1}^{n} Y_{t}^{j} d t+\lambda \sum_{j=1}^{n} d Y_{t}^{j}+\lambda d Y_{t}^{i}-\gamma Y_{t}^{i} d t=0 .
$$

The $n$ such equations for each trader can be collected together as

$$
\lambda\left(I+\mathbf{1 1}^{T}\right) d Y_{t}=\gamma\left(I-\mathbf{1 1}^{T}\right) Y_{t} d t,
$$

where $I$ is the $n \times n$ identity matrix, $\mathbf{1}$ is the $n$-vector with all entries equal to one, and $\mathbf{1 1}^{T}$ is an $n \times n$ matrix with all elements equal to one. From the formula for the inverse of the rank-one update of a matrix (Kailath (1980)), the inverse of $I+\mathbf{1 1}^{T}$ is $I-\frac{1}{n+1} \mathbf{1 1}^{T}$, which we use to write the linear dynamic system in the form

$$
d Y_{t}=\frac{\gamma}{\lambda} A Y_{t} d t, \quad \text { where } \quad A=I-\frac{2}{n+1} \mathbf{1 1}^{T} .
$$

Since $A \mathbf{1}=\mathbf{1}-\frac{2}{n+1} n \mathbf{1}=-\frac{n-1}{n+1} \mathbf{1}$, the vector of ones is an eigenvector of the matrix $A$, with associated eigenvalue $-\frac{n-1}{n+1}$. Likewise, vectors in the null-space of 1 are eigenvectors of $A$, with eigenvalue 1: For $v$ orthogonal to the vector of ones, that is, satisfying $\mathbf{1}^{T} v=0$, we find that $A v=v$. The dimension of this subspace, and multiplicity of the eigenvalue 1 , is $n-1$. Since the matrix $A$ has a full set of $n$ independent eigenvectors, all Jordan blocks are of size one and solutions to the system of linear differential equations are as stated in (5). This characterizes any continuous policy (with continuous dual functional) that is an extremal of the problem. Since a continuous extremal exists, from the concavity of the objective in the state and control and from the linearity of the constraint, this is the unique extremal of the problem (in the admissible class). The $n$ trading target constraints and $\mathbf{1}^{T} b=0$ uniquely determine the $n$ free parameters in the solution (integrate the $Y_{t}^{i}$, equate to $\Delta x_{i}$, and solve for $a$ and $b$ ). Q.E.D. 
We next show a lemma and corollary, which will be of use in proving Result 2.

Lemma A.1: The function $f: \mathbf{R}^{+} \mapsto \mathbf{R}$

$$
f(y)=\frac{1+e^{-y}}{1-e^{-y}}-\frac{2}{y}
$$

is positive increasing.

Proof: We first show $\lim _{y \rightarrow 0} f(y)=0$. Applying l'Höpital's rule, we find

$$
\lim _{y \rightarrow 0} f(y)=\lim _{y \rightarrow 0} \frac{-y e^{-y}+1+e^{-y}-2 e^{-y}}{1-e^{-y}+y e^{-y}}=\lim _{y \rightarrow 0} \frac{y e^{-y}}{2 e^{-y}-y e^{-y}}=0 .
$$

We now show $f^{\prime}(y)>0$ :

$$
f^{\prime}(y)=\frac{-2 e^{-y}}{\left(1-e^{-y}\right)^{2}}+\frac{2}{y^{2}}=\frac{2}{\left(1-e^{-y}\right)^{2} y^{2}}\left(-y^{2} e^{-y}+1+e^{-2 y}-2 e^{-y}\right) .
$$

For $y>0$, the denominator is positive. We show that the numerator is also positive, $g(y)=-y^{2} e^{-y}+1+e^{-2 y}-2 e^{-y}>0$, from $g(0)=0$ and $g^{\prime}(y)>0$ :

$$
g^{\prime}(y)=-2 y e^{-y}+y^{2} e^{-y}-2 e^{-2 y}+2 e^{-y}=e^{-y}\left(-2 y+y^{2}-2 e^{-y}+2\right) .
$$

Likewise, we show $h(y)=-2 y+y^{2}-2 e^{-y}+2>0$, from $h(0)=0$ and $h^{\prime}(y)>0$ :

$$
h^{\prime}(y)=-2+2 y+2 e^{-y},
$$

which is positive if $e^{-y}>1-y$, which is true for any $y \neq 0$ (from the intercept and derivative at zero and from the convexity of the exponential). Q.E.D.

Corollary A.1: The function $g: \mathbf{R}^{+} \mapsto \mathbf{R}$

$$
g(y)=y \frac{1+e^{-y}}{1-e^{-y}}
$$

is positive increasing.

Proof: Write

$$
g(y)=y f(y)+2,
$$

where $f$ is as in the previous lemma. The product of two positive increasing functions is positive increasing. Q.E.D.

Proof of Result 2 (Expected total surplus and loss for multiple traders): The expected surplus is obtained by integration of

$$
-P_{t} \sum_{i=1}^{n} Y_{t}^{i}=-P_{t} n a e^{-\frac{n-1}{n+1} \frac{\gamma}{\lambda} t}
$$


over $t \in[0, T]$, followed by algebraic simplification. The proofs of the monotonicities are direct applications of the lemma above or of its corollary, using $y=T, y=\gamma, y=\frac{1}{\lambda}$, and $y=\frac{n-1}{n+1}$ (with $n$ relaxed to be in $\mathbf{R}$ ). For $\lambda$ and $n$, we also need the fact that the composition of two monotonic functions is monotonic. Q.E.D.

Proof of Result 3 (Expected total surplus and loss for two traders): By equation (8), $Y=Y_{t}^{d}+Y_{t}^{p}=2 a e^{-\frac{1}{3} \frac{\gamma}{\lambda} t}$. By integration of $-P_{t} Y$ over $t \in[0, T]$, followed by algebraic simplification, the results in equations (13) and (16) are derived. The monotonicities are verified by differentiation of equation (16). We define $V_{-}=V_{d}-V_{p}$ and $Y_{-}=Y_{t}^{d}-Y_{t}^{p}=2 b e^{\frac{\gamma}{\lambda} t}$. Integrate $-P_{t} Y_{-}$over $t \in[0, T]$ and simplify to obtain

$$
V_{-}=V_{d}-V_{p}=-u \Delta x-\gamma \frac{e^{\frac{\gamma}{\lambda} T}}{e^{\frac{\gamma}{\lambda} T}-1} \Delta x^{2} .
$$

Then $V_{d}$ and $V_{p}$ are obtained by simplification of $\left(V_{2}+V_{-}\right) / 2$ and $\left(V_{2}-V_{-}\right) / 2$.

The proof for the monotonicity of $\frac{V_{p}}{\Delta V_{d}}$ is along the same lines as for Result 2 (with lengthier algebra). The bounds on $\frac{V_{p}}{\Delta V_{d}}$ are the limits at 0 and $+\infty$, obtained by applying l'Höpital's rule as needed. Q.E.D.

Proof of Result 4 (Repeated game with two symmetric traders): For trader 1, the gains from cooperation must exceed those of one-time deviation and infinite noncooperation, or

$$
\begin{aligned}
& \frac{\delta_{1}}{1-\delta_{1}}\left[p_{1}\left(1-p_{2}\right) V_{1}(\Delta x)+\frac{1}{2} p_{1} p_{2} V_{1}(2 \Delta x)\right] \\
& \quad \geq V_{p}(\Delta x)+\frac{\delta_{1}}{1-\delta_{1}}\left[\left(1-p_{1}\right) p_{2} V_{p}(\Delta x)+p_{1}\left(1-p_{2}\right) V_{d}(\Delta x)+\frac{1}{2} p_{1} p_{2} V_{2}(2 \Delta x)\right] .
\end{aligned}
$$

Since $\Delta V_{2}$ is quadratic in $\Delta x$, we have that $\frac{1}{2} \Delta V_{2}(2 \Delta x)=2 \Delta V_{2}(\Delta x)$ (we then omit the argument when it is $\Delta x$ ). The first equation in (19) follows by solving for $\delta_{1}$. The second equation is derived similarly for trader two. For both traders to cooperate, it must be that $\delta \geq \max \left\{\delta_{1}, \delta_{2}\right\}$. The monotonicities can be proved algebraically, along the same lines as for Result 2. Q.E.D.

Proof of Result 5 (Shocks of random magnitude): Equation (A15) can be written in this context as equation (26), where $f$ is the density of $\Delta x$, and $C$ and $K$ are as defined. For values of $C$ sufficiently large or values of $K$ sufficiently small, equation (26) will be satisfied and there will exist a $\Delta x$ such that cooperation is possible. As long as $|\Delta x|<\Delta x^{*}$, the traders will cooperate since the value of cooperating exceeds that of a one-time deviation and subsequent grimtrigger play. If $|\Delta x| \geq \Delta x^{*}$, the traders will predate and resume cooperation in the next period if possible. If equation (26) is not satisfied, then cooperation is not possible and the traders will always predate. Thus, there exists a 
subgame-perfect Nash equilibrium as described. The left-hand side of equation (26) is bounded since $f$ has finite variance and the right-hand side is unbounded. Hence, the supremum of $\Delta x^{*}$ is bounded. For existence of $\overline{\Delta x}$, note that zero is a solution. Q.E.D.

\section{Appendix B}

\section{Closed-Loop Solution}

In our analysis of the stage game, the strategies considered are deterministic. They are open-loop, in the sense that traders choose their strategies at time $t=0$. It is assumed that traders would not respond to other traders' deviations from their optimal strategies. We would have obtained the same solution had we defined the $Y_{t}^{i}$ to be $\mathcal{F}_{t}$-adapted (where $\mathcal{F}_{t}$ is the filtration of the $\sigma$-algebras generated by $B_{t}$ ). Note, however, that this is in the strict sense that traders are aware of the underlying process $U_{t}$ that defines price fundamentals, but not of the actual price $P_{t}$ and of the other players' trading rates $Y_{t}$. (The argument for the solution to this variation on the open-loop problem to be the same as in Result 1 is based on the value functions' linearity in $U_{t}$, as is done below in this section.)

We now consider closed-loop strategies, in that traders know or can infer the other traders' rate of trading and respond accordingly. Under such strategies, $Y_{t}^{i}$ is adapted to all the information existing at time $t$, and players can revise their trading decisions at any time based on such information. This means that earlier decisions must account for other players' response function at later times. This inability to commit ahead of time to not deviate from a given strategy over the entire $[0, T]$ period leads to more aggressive strategies than in the open-loop case, including faster racing.

Closed-loop strategies are substantially more difficult to analyze than openloop strategies, and thus we are not able to provide a closed-form solution. We do provide a description of the equilibrium optimal strategies in terms of a value function with two scalar parameters that satisfy a triangular system of nonlinear differential equations. Our numerical simulations show that the open- and closed-loop solutions are not substantially different. We provide some analytical justification for this observation. In particular, from a fixed-point analysis of the Riccati equations, we provide closed-form expressions for the expected value for each of the players when $T$ is large.

We derive the closed-loop result for the deterministic case and then show that it holds for the stochastic pricing equation. We consider strategies in which the trading rate is constant over time increments of length $\Delta t$. The reward for trader $i$ over each time increment is

$$
\begin{aligned}
r_{i}(u, y, \Delta t) & =\int_{0}^{\Delta t}-\left(u+\gamma \mathbf{1}^{T} y \tau+\lambda \mathbf{1}^{T} y\right) y_{i} d \tau \\
& =-\left(u+\left(\frac{\gamma}{2} \Delta t+\lambda\right) \mathbf{1}^{T} y\right) y_{i} \Delta t
\end{aligned}
$$


We formulate the problem as a dynamic game, with an $n+1$-dimensional state, composed of $u(t) \in \mathbf{R}$ and $\phi(t) \in \mathbf{R}^{n}$. The first component, $u(t)$, is the expected price at time $t$, including the permanent price impact of previous trades (in previous notation, $\left.u(t)=U_{t}+\gamma \sum_{i=1}^{n} X_{t}^{i}\right)$. The $n$ components of $\phi(t)$ are the remaining trading targets for each trader, that is, the amount they still need to trade by $T$ (in previous notation, $\phi_{i}(t)=x_{T i}-X_{t}^{i}$ ). The state transition over a period of length $\Delta t$ with each player trading at a constant rate $y_{i}$ is

$$
\begin{aligned}
& u^{\prime}(u, y, \Delta t)=u+\gamma \mathbf{1}^{T} y \Delta t, \\
& \phi^{\prime}(\phi, y, \Delta t)=\phi-y \Delta t .
\end{aligned}
$$

The value functions for each of the $n$ traders must simultaneously satisfy

$$
\begin{array}{r}
V^{i}(u, \phi, t)=\max _{y_{i}} r_{i}(u, y, \Delta t)+V^{i}\left(u^{\prime}(u, y, \Delta t), \phi^{\prime}(\phi, y, \Delta t), t+\Delta t\right), \\
i=1, \ldots, n .
\end{array}
$$

We show that the value functions can be represented in the form

$$
V^{i}(u, \phi, t)=-u \phi_{i}-\alpha(t) \phi_{i} \mathbf{1}^{T} \phi+\beta(t)\left(\mathbf{1}^{T} \phi\right)^{2},
$$

and derive Riccati equations for $\alpha(t), \beta(t) \in \mathbf{R}$. We take the limit $\Delta t \rightarrow 0$ to find the differential equations for the continuous-time case.

Substituting equations (B6), (B2), (B3), and (B4) in equation (B5) and letting $\Delta t \rightarrow 0$, we obtain

$$
\begin{aligned}
& -\frac{d \alpha(t)}{d t} \phi_{i} \mathbf{1}^{T} \phi+\frac{d \beta(t)}{d t}\left(\mathbf{1}^{T} \phi\right)^{2} \\
& \quad=\max _{y_{i}}-\lambda y_{i} \mathbf{1}^{T} y-\gamma \phi_{i} \mathbf{1}^{T} y+\alpha \phi_{i} \mathbf{1}^{T} y+\alpha y_{i} \mathbf{1}^{T} \phi-2 \beta \mathbf{1}^{T} y \mathbf{1}^{T} \phi .
\end{aligned}
$$

Differentiating with respect to $y_{i}$ and equating to zero, we obtain the optimality condition

$$
\lambda\left(y_{i}+\mathbf{1}^{T} y\right)=(\alpha-\gamma) \phi_{i}+(\alpha-2 \beta) \mathbf{1}^{T} \phi,
$$

which we collect over $i=1, \ldots, n$ as

$$
\lambda\left(I+\mathbf{1 1}^{T}\right) y=\left((\alpha-\gamma) I+(\alpha-2 \beta) \mathbf{1 1}^{T}\right) \phi .
$$

Multiplying on the left by $\frac{1}{\lambda}\left(I-\frac{1}{n+1} \mathbf{1 1}^{T}\right)$, we solve for the equilibrium trading rates as a function of the state,

$$
y=\frac{1}{\lambda}\left((\alpha-\gamma) I+\frac{1}{n+1}(-2 \beta+\gamma) \mathbf{1 1}^{T}\right) \phi,
$$

which can be written individually as

$$
y_{i}=\frac{1}{\lambda}\left((\alpha-\gamma) \phi_{i}+\frac{1}{n+1}(-2 \beta+\gamma) \mathbf{1}^{T} \phi\right), \quad i=1, \ldots, n .
$$


The sum of the trading rates is then

$$
\mathbf{1}^{T} y=\frac{1}{\lambda}\left(\alpha-2 \frac{n}{n+1} \beta-\frac{1}{n+1} \gamma\right) \mathbf{1}^{T} \phi .
$$

Substituting equations (B11) and (B12) in equation (B7), we verify that the structure of the value function as postulated in (B6) is indeed preserved over time (more strongly, it can be verified that the structure is preserved before letting $\Delta t \rightarrow 0$ ). Collecting terms and simplifying, we obtain the following triangular representation for the Riccati equations,

$$
\begin{aligned}
& \frac{d \alpha}{d t}=\frac{1}{\lambda} \alpha(\alpha-\gamma), \\
& \frac{d \beta}{d t}=\frac{1}{\lambda}\left(2 \alpha \beta-\frac{1}{(n+1)^{2}}(2 n \beta+\gamma)^{2}\right) .
\end{aligned}
$$

So far we have assumed a deterministic $u$. The objectives for the deterministic case are linear in $u$, the corresponding optimal policies do not depend on $u$, and the value functions were found to be linear in $u$. Using these value functions for the stochastic case, the $\partial^{2} V^{i} / \partial u^{2}$ terms in the HJB equations are zero, and all other terms are as in the HJB equations for the deterministic case. That is, if given value functions and optimal policies satisfy the HJB equations for the deterministic case, they also satisfy the HJB equations for the stochastic case.

Conditions for verification of the HJB equation are met (Karatzas and Shreve (1988)) as the value function is differentiable in $t$ and smooth in the state.

While the system of nonlinear equations (B13) and (B14) is difficult to solve in closed form, a number of its properties can be studied. As $T \rightarrow \infty$, since the expected values are bounded due to the overall convexity of the problem and the triangular structure precludes oscillatory behavior, $\alpha(0)$ and $\beta(0)$ must converge to fixed points of the differential equations. The fixed points for the first equation are 0 and $\gamma$, of which only $\alpha=\gamma$ is stable (note that equation(B13) is convex-quadratic in $\alpha$, and we are considering integration backwards in time). With $\alpha=\gamma$, we solve for the fixed points of $\beta$, which are found to be $\frac{1}{2} \gamma$ and $\frac{1}{2 n^{2}} \gamma$, of which only $\beta=\frac{1}{2 n^{2}} \gamma$ is stable (note that equation (B14) is concave-quadratic in $\beta$ ).

In the case $n=1$, we have $\phi_{i}(0) \mathbf{1}^{T} \phi(0)=\left(\mathbf{1}^{T} \phi(0)\right)^{2}=\Delta x^{2}$, so that

$$
V=-U_{0} \Delta x-\gamma \Delta x^{2}+\frac{1}{2} \gamma \Delta x^{2}=-U \Delta x-\frac{1}{2} \gamma \Delta x^{2} .
$$

As expected, we recover the same value as for the open-loop case (which corresponds to a constant trading rate).

Consider now the case $n=2$, with the distressed trader needing to trade $\phi_{1}(0)=\Delta x$, and the predatory trader's target being $\phi_{2}(0)=0$. For the distressed trader, $\phi_{1}(0) \mathbf{1}^{T} \phi(0)=\left(\mathbf{1}^{T} \phi(0)\right)^{2}=\Delta x^{2}$. For the predatory trader, $\phi_{2}(0) \mathbf{1}^{T} \phi(0)=$ 0 and $\left(\mathbf{1}^{T} \phi(0)\right)^{2}=\Delta x^{2}$. Table AI summarizes the same expected values as in Table I, but for $T$ large ( $T \rightarrow \infty$, or equivalently, by change of units, $\lambda$ small, 


\section{Table AI}

\section{Expected Values from Strategic Trading: Comparing Open-Loop and Closed-Loop Strategies}

Listed are the expected values that traders gain or lose when a sell-off occurs in the market. Considering the limit conditions when $T \rightarrow \infty$ or $\lambda \rightarrow 0$, we calculate the values to the strategic traders when they use open-loop and closed-loop strategies. $U_{0}$ is the expected value of future dividends and $\gamma$ measures the permanent impact of trading. The values to the traders are qualitatively similar, though the aggregate surplus loss to the traders is worse in the closed-loop case.

\begin{tabular}{|c|c|c|c|}
\hline & & Open-Loop & Closed-Loop \\
\hline $\begin{array}{l}\text { Surplus to distressed trader, no } \\
\text { predation }\end{array}$ & $V_{1}$ & $-U_{0} \Delta x-\frac{1}{2} \gamma \Delta x^{2}$ & $-U_{0} \Delta x-\frac{1}{2} \gamma \Delta x^{2}$ \\
\hline $\begin{array}{l}\text { Surplus to distressed trader } \\
\text { during predation }\end{array}$ & $V_{d}$ & $-U_{0} \Delta x-\frac{5}{6} \gamma \Delta x^{2}$ & $-U_{0} \Delta x-\frac{7}{8} \gamma \Delta x^{2}$ \\
\hline $\begin{array}{l}\text { Change in surplus to distressed } \\
\text { trader }\left(V_{1}-V_{d}\right)\end{array}$ & $\Delta V_{d}$ & $\frac{1}{3} \gamma \Delta x^{2}$ & $\frac{3}{8} \gamma \Delta x^{2}$ \\
\hline Surplus to predator & $V_{p}$ & $\frac{1}{6} \gamma \Delta x^{2}$ & $\frac{1}{8} \gamma \Delta x^{2}$ \\
\hline $\begin{array}{l}\text { Total surplus to predator and } \\
\text { distressed trader }\end{array}$ & $V_{2}$ & $-U_{0} \Delta x-\frac{2}{3} \gamma \Delta x^{2}$ & $-U_{0} \Delta x-\frac{3}{4} \gamma \Delta x^{2}$ \\
\hline $\begin{array}{l}\text { Change in overall surplus with } \\
\text { predatory trading }\end{array}$ & $\Delta V_{2}$ & $\frac{1}{6} \gamma \Delta x^{2}$ & $\frac{1}{4} \gamma \Delta x^{2}$ \\
\hline
\end{tabular}

i.e., $\lambda \rightarrow 0$ ). Overall, the expected values are similar. The loss to the distressed trader is somewhat larger in the closed-loop case, the gain to predatory trader somewhat smaller, and the overall welfare loss somewhat larger. The ratio of gains to the predatory trader per losses to the distressed trader decreases from $1 / 2$ in the open-loop case to $1 / 3$ in the closed-loop case (see lower bound in Result 3). Cooperation between traders will therefore be more likely in the closed-loop case.

For $n$ traders, and still under the assumption of a long trading horizon, we can derive the shape of the racing behavior under closed-loop strategies. During the racing stage, that is, for small $t, \alpha(t)$ and $\beta(t)$ are approximately constant (under the assumption of large $T$ or small $\lambda$ ). Using the stable fixed-point values $\alpha=\gamma$ and $\beta=\frac{1}{2 n^{2}} \gamma$ in equation (B11), we obtain

$$
y_{i}=\frac{\gamma}{\lambda} \cdot \frac{n-1}{n} \cdot \frac{\mathbf{1}^{T} \phi}{n}, \quad i=1, \ldots, n
$$

Since $y_{i}=-d \phi_{i} / d t$, we conclude that with closed-loop strategies, the racing behavior is of the form

$$
y_{i}=a e^{-\frac{\gamma}{\lambda} \frac{n-1}{n} t}
$$

with the constant $a \in \mathbf{R}$ a function of the average trading target. This is slightly faster than what we find for the open-loop case, $y_{i}=a e^{-\frac{\gamma}{\lambda} \frac{n-1}{n+1} t}$. 


\section{REFERENCES}

Abreu, Dilip, 1988, On the theory of infinitely repeated games with discounting, Econometrica 56, 383-396.

Abreu, Dilip, David Pearce, and Ennio Stacchetti, 1986, Optimal cartel equilibria with imperfect monitoring, Journal of Economic Theory 39, 251-269.

Acharya, Viral, and Lasse Pedersen, 2005, Asset pricing with liquidity risk, Journal of Financial Economics 77, 375-410.

Almgren, Robert, 2000, Optimal execution of portfolio transactions, Journal of Risk 3, 5-39.

Almgren, Robert, and Neil Chriss, 1999, Value under liquidation, Risk 12, 61-63.

Attari, Mukarram, Antonio Mello, and Martin Ruckes, 2005, Arbitraging arbitrageurs, Journal of Finance 60, 2471-2511.

Back, Kerry, and Shmuel Baruch, 2004, Information in securities markets: Kyle meets Glosten and Milgrom, Econometrica 72, 433-465.

Basar, Tomas, and Geert Olsder, 1999, Dynamic Noncooperative Game Theory (Society for Industrial and Applied Mathematics: Philadelphia).

Battalio, Robert, Andrew Ellul, and Robert Jennings, 2007, Reputation effects in trading on the New York Stock Exchange, Journal of Finance 62, 1243-1271.

Berhardt, Dan, Vladimir Dvoracek, Eric Hughson, and Ingrid Werner, 2005, Why do larger orders receive discounts on the London stock exchange? Review of Financial Studies 18, 1343-1368.

Bernheim, Douglas, and Michael Whinston, 1990, Multimarket contact and collusive behavior, RAND Journal of Economics 21, 1-26.

Bertsimas, Dimitris, and Andrew Lo, 1998, Optimal control of execution costs, Journal of Financial Markets 1, 1-50.

Brunnermeier, Markus, and Lasse Pedersen, 2005, Predatory trading, Journal of Finance 60, 18251863.

Chan, Louis, and Josef Lakonishok, 1995, The behavior of stock prices around institutional trades, Journal of Finance 50, 1147-1174.

Cheng, Minder, and Ananth Madhavan, 1997, In search of liquidity: Block trades in the upstairs and downstairs markets, Review of Financial Studies 10, 175-203.

Cocco, Joao, Francisco Gomes, and Nuno Martins, 2003, Lending relationships in the interbank market, Working paper, London School of Business.

DeMarzo, Peter, and Branko Uroevic, 2000, Optimal trading by a large shareholder, Working paper, Stanford Graduate School of Business and Haas School of Business.

Desgranges, Gabriel, and Thierry Foucault, 2005, Reputation-based pricing and price improvements in dealership markets, Journal of Economics and Business 57, 493-527.

Duffie, Darrell, Nicolae Garleanu, and Lasse Pedersen, 2005, Over-the-counter markets, Econometrica 73, 1815-1847.

Dutta, Prajit, and Ananth Madhavan, 1997, Competition and collusion in dealer markets, Journal of Finance 52, 245-276.

Fedyk, Yuriy, 2001, Large investor optimization problem with direct effect of trades on prices, Working paper, Olin School of Business.

Foster, F. Douglas, and S. Viswanathan, 1996, Strategic trading when agents forecast the forecasts of others, Journal of Finance 51, 1437-1478.

Friedman, James, 1971, A non-cooperative equilibrium for supergames, Review of Economic Studies $38,1-12$.

Gennotte, Gerard, and Albert Kyle, 1991, Intertemporal insider trading with a smooth order flow, Working paper, Haas School of Business Administration, Berkeley.

Green, Edward, and Robert Porter, 1984, Noncooperative collusion under imperfect price information, Econometrica 52, 87-100.

Hansch, Oliver, Narayan Naik, and S. Viswanathan, 1999, Preferencing, internalization, best execution, and dealer profits, Journal of Finance 54, 1799-1828.

Holthausen, Robert, Richard Leftwich, and David Mayers, 1990, Large block transactions, the speed of response, and temporary and permanent stock-price effects, Journal of Financial Economics 26, 71-95. 
Huang, Roger, and Hans Stoll, 1997, The components of the bid-ask spread: A general approach, Review of Financial Studies 10, 995-1034.

Huberman, Gur, and Werner Stanzl, 2004a, Arbitrage-free price update and price-impact functions, Econometrica 72, 1247-1275.

Huberman, Gur, and Werner Stanzl, 2004b, Optimal liquidity trading, Review of Finance 9, 165200.

Kailath, Thomas, 1980, Linear Systems (Prentice-Hall, New Jersey).

Karatzas, Ioannis, and Steven Shreve, 1988, Brownian Motion and Stochastic Calculus (Springer, Berlin).

Kaul, Aditya, Vikas Mehrotra, and Randall Morck, 2000, Demand curves for stocks do slope down: New evidence from an index weights adjustment, Journal of Finance 55, 893-912.

Keim, Donald, and Ananth Madhavan, 1996, The upstairs market for large-block transactions: Analysis and measurement of price effects, Review of Financial Studies 9, 1-36.

Kraus, Alan, and Hans Stoll, 1972, Price impacts of block trading on the New York Stock Exchange, Journal of Finance 27, 569-588.

Massa, Massimo, and Andrei Simonov, 2003, Reputation and interdealer trading: A microstructure analysis of the treasury bond market, Journal of Financial Markets 6, 99-141.

Pástor, Ľuboš, and Robert Stambaugh, 2003, Liquidity risk and expected stock returns, Journal of Political Economy 111, 642-685.

Pritsker, Matthew, 2004, Large investors: Implications for equilibrium asset returns, shock absorption, and liquidity, Working paper, Federal Reserve, Washington D.C.

Ramadorai, Tarun, 2003, Persistence, performance, and prices in foreign exchange markets, Working paper, Harvard University.

Reiss, Peter, and Ingrid Werner, 2004, Anonymity, adverse selection and the sorting of interdealer trades, Review of Financial Studies 18, 599-636.

Rotemberg, Julio, and Garth Saloner, 1986, A supergame-theoretic model of price wars during booms, American Economic Review 76, 390-407.

Sadka, Ronnie, 2006, Momentum and post-earnings-announcement drift anomalies: The role of liquidity risk, Journal of Financial Economics, 80, 309-349.

Seppi, Duane, 1990, Equilibrium block trading, Journal of Finance 45, 73-94.

Vayanos, Dimitri, 1998, Transaction costs and asset prices: A dynamic equilibrium model, Review of Financial Studies 11, 1-58. 